\title{
Quantitative ultrasound imaging of soft biological tissues: a primer for radiologists and medical physicists
}

\author{
Guy Cloutier ${ }^{1,2,3^{*}} \mathbb{D}$, François Destrempes ${ }^{1}$, François $Y_{u^{2,3,4}}$ and An Tang ${ }^{2,5,6}$
}

\begin{abstract}
Quantitative ultrasound (QUS) aims at quantifying interactions between ultrasound and biological tissues. QUS techniques extract fundamental physical properties of tissues based on interactions between ultrasound waves and tissue microstructure. These techniques provide quantitative information on sub-resolution properties that are not visible on grayscale (B-mode) imaging. Quantitative data may be represented either as a global measurement or as parametric maps overlaid on B-mode images. Recently, major ultrasound manufacturers have released speed of sound, attenuation, and backscatter packages for tissue characterization and imaging. Established and emerging clinical applications are currently limited and include liver fibrosis staging, liver steatosis grading, and breast cancer characterization. On the other hand, most biological tissues have been studied using experimental QUS methods, and quantitative datasets are available in the literature. This educational review addresses the general topic of biological soft tissue characterization using QUS, with a focus on disseminating technical concepts for clinicians and specialized QUS materials for medical physicists. Advanced but simplified technical descriptions are also provided in separate subsections identified as such. To understand QUS methods, this article reviews types of ultrasound waves, basic concepts of ultrasound wave propagation, ultrasound image formation, point spread function, constructive and destructive wave interferences, radiofrequency data processing, and a summary of different imaging modes. For each major QUS technique, topics include: concept, illustrations, clinical examples, pitfalls, and future directions.
\end{abstract}

Keywords: Quantitative ultrasound imaging, Speed of sound imaging, Ultrasound attenuation imaging, Backscatter imaging, Backscatter coefficient

\section{Key points}

- Quantitative ultrasound (QUS) provides images on interactions between ultrasound waves and biological tissues. - Tissue-specific speed of sound images can be produced for QUS tissue characterization.
- Acoustic attenuation is used in QUS as biomarkers to produce images independent of ultrasound system characteristics and settings.

- Backscatter coefficient is independent of ultrasound system characteristics and settings for tissue characterization.

\footnotetext{
*Correspondence: guy.cloutier@umontreal.ca

${ }^{1}$ Laboratory of Biorheology and Medical Ultrasonics, Centre de recherche

du Centre hospitalier de I'Université de Montréal (CRCHUM), 900 St-Denis,

Montréal, Québec H2X 0A9, Canada

${ }^{2}$ Department of Radiology, Radio-oncology, and Nuclear Medicine,

Université de Montréal, Montréal, Québec, Canada.

${ }^{3}$ Institute of Biomedical Engineering, Université de Montréal, Montréal,

Québec, Canada.

Full list of author information is available at the end of the article
}

\section{Introduction}

The field of quantitative ultrasound (QUS) imaging has been active for more than 50 years and it is only recently that ultrasound manufacturers have started implementing some of these biomarkers on clinical scanners. Several technical textbooks have described state-of-the-art 
innovations [1-4]. Interested readers may refer to a recent contribution for a thorough introduction to the field of QUS imaging [4]. Technical constraints such as the need to perform an additional acquisition on a reference phantom and measurement variability have delayed clinical adoption [5]. However, the development of dedicated instruments using QUS methods for the assessment of bone structures [6] and liver steatosis [7] has led to a resurgence of the field of QUS. Recently, major ultrasound radiology manufacturers have released speed of sound, attenuation, and backscatter packages for tissue characterization and imaging.

In contrast to brightness (B-mode) grayscale ultrasound imaging that provides qualitative information on anatomy, QUS aims at quantifying physical phenomena associated with the propagation of ultrasound into biological tissues. More specifically, QUS extracts fundamental properties of a tissue based on the interactions of propagating ultrasound waves with the tissue microstructure. These ultrasound sub-resolution quantitative signatures of the tissue microstructure are then used to produce a measurement of a global physical quantity within a region of interest (ROI) or parametric images for diagnosis. As illustrated in Fig. 1, these images are complementary to grayscale imaging, Doppler approaches measuring flow and tissue motion, and elastography (strain and shear wave based) assessing mechanical properties of tissues. While grayscale imaging, Doppler imaging, and elastography can also provide quantitative measures, the use of the terminology "quantitative" in QUS refers to a specific field dedicated to biomarkers describing wave interactions with the insonified organ.

The field of QUS imaging was initially described as "ultrasound tissue characterization (UTC)" [8]. Following the first international seminar on tissue characterization by ultrasound held in 1975 at the National Bureau of Standards in Washington, UTC was enthusiastically identified "to be one of the next big developments in the clinical utilization of ultrasound" (see this Editorial statement in [8]). After several decades of research marked

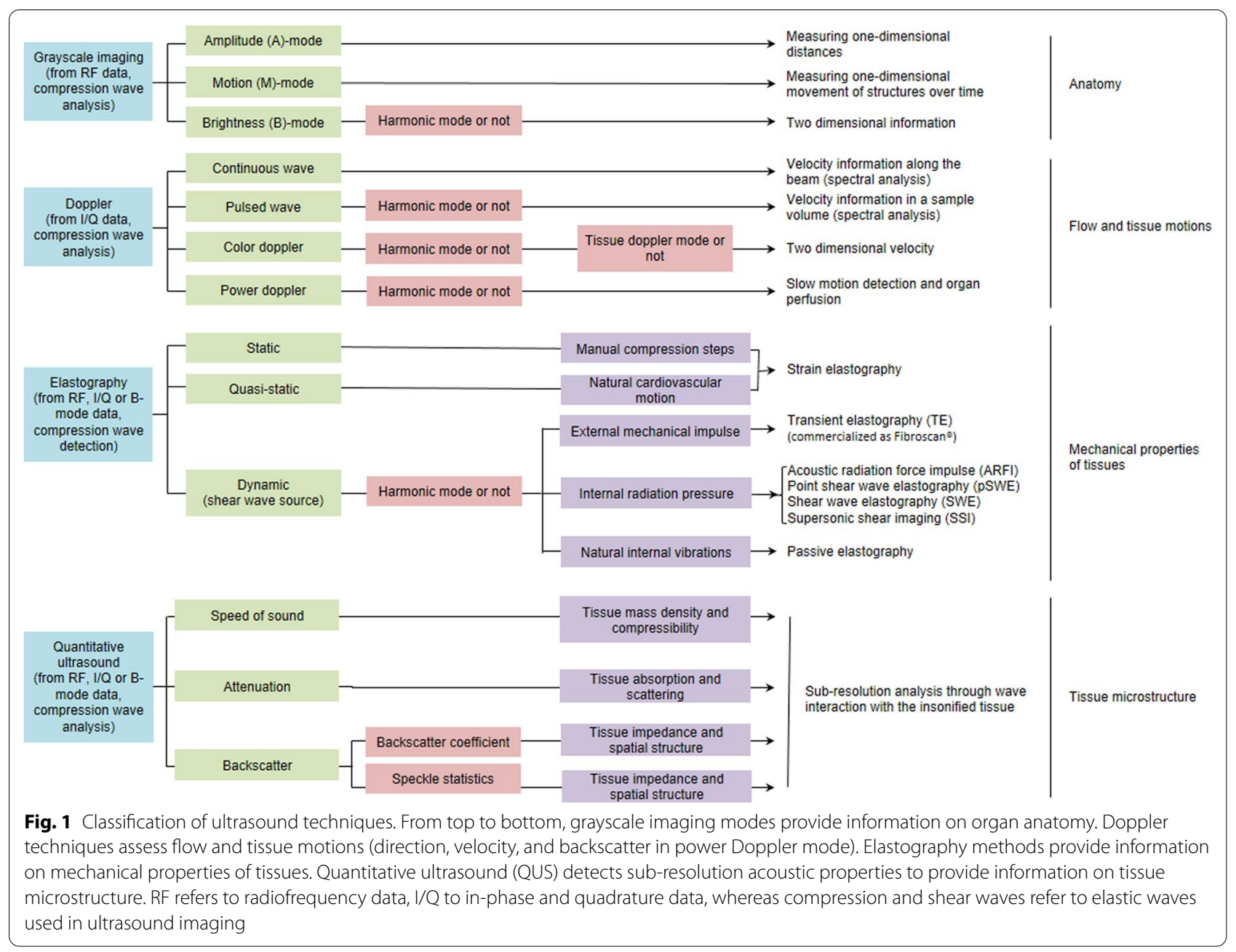


by an unsuccessful translation of UTC technologies to ultrasound manufacturers and clinical practice, technical scientists strategically started introducing the concept of QUS, which in fact is in line with UTC initial developments aiming at providing biomarkers based on the interactions of ultrasound waves with the underlying tissue. QUS was first defined as a specific research field to characterize bone structures using attenuation and speed of sound measurements $[9,10]$. UTC imaging is still used today to define this field of research [11], but it is no longer specific to QUS technologies based on the physics of wave interactions with biological tissues [12]. Indeed, UTC also refers to image processing methods to extract tissue information.

In this article, we refer to QUS measurements or images with a focus on soft tissue characterization, and on examples of QUS methods applied to grade liver steatosis and breast cancer (because those applications are the first ones using implementations of QUS on clinical scanners). Basic concepts behind speed of sound, attenuation, and backscatter measurements to grade a normal or a pathological state of an organ are reviewed. We describe key QUS technical concepts and illustrate QUS methods by avoiding complex mathematical equations. We provide a glossary of key terms relevant to this field (Table 1). To understand QUS methods, this article reviews basic concepts on the type of acoustic waves, speed of sound of these waves, ultrasound wave propagation phenomena, image formation, point spread function, constructive and destructive wave interferences, and radiofrequency data processing versus B-mode images. For each QUS method, topics include simplified concepts, units, range of values for soft biological tissues, measurement methods, illustrations, clinical examples, pitfalls, and future directions. For a few technical descriptions, the label "advanced materials" is used and the text is in italic.

\section{Background on ultrasound wave propagation and image processing}

Types of acoustic waves

In fundamental acoustics, different types of mechanical waves, called elastic waves, can propagate into soft biological tissues. Among those, compression and shear waves are currently used on clinical ultrasound scanners (Fig. 2). Compression waves, also known as longitudinal waves, consist in alternating compressions and dilations of the tissue, where the direction of wave propagation is parallel to the direction of the source. Compression waves are used in all imaging modes (grayscale, Doppler, elastography, and QUS). Shear waves, also known as transverse waves, consist in alternating shearing of the tissue, where the oscillation motion is perpendicular to the direction of the wave propagation. Besides compression and shear waves, other types of elastic waves can travel into biological tissues as surface waves (i.e., Rayleigh and Love waves) $[13,14]$. The latter types of waves are being investigated for ultrasound imaging research and developments $[15,16]$.

\section{Speed of sound}

The speed of sound of compression waves is fixed on most ultrasound scanners to $1,540 \mathrm{~m} / \mathrm{s}$, and this value is used to convert time to distance for producing an image. Indeed, ultrasound systems are measuring echo times between emission and reception, and a fixed speed of sound is assumed to map received echoes into spatial depth. In reality, the actual speed of sound varies depending on tissue structural characteristics. This assumption produces errors as the real speed of sound in soft tissues varies by $\pm 150 \mathrm{~m} / \mathrm{s}$ or about $10 \%$ of the assumed speed of sound [17, 18]. In QUS, tissue-dependent variations in speed of sound are retrieved and used as a biomarker of a pathological state. Shear waves are much slower and travel at a speed of a few $\mathrm{m} / \mathrm{s}$ in biological tissues (typically $<10 \mathrm{~m} / \mathrm{s}$ ). In shear wave elastography, the shear wave speed is used to infer on tissue elasticity. Rayleigh and Love waves, which will not be further discussed, are also moving at a speed of a few $\mathrm{m} / \mathrm{s}$ in biological tissues (typically $<10 \mathrm{~m} / \mathrm{s}$ ).

Advanced materials: The speed of a compression wave is determined by the square root of the bulk elasticity modulus divided by the mass density of the tissue. The bulk modulus, or isostatic elasticity modulus, is the constant of proportionality relating the stress-strain linear behavior of a tissue deformed by a compressive movement or a compression wave in the context of this review. We recall here that ultrasound systems are considering a constant bulk modulus and a constant mass density to produce depth information on images (i.e., a constant speed of sound). However, it is the change in speed of sound at interfaces that allows observing image boundaries and tissue contrast. In other words, a constant speed of sound is required to estimate distances on the image but without speed of sound heterogeneities, no image would be produced. Notice that the concept of adiabatic compressibility can be found in the technical ultrasound literature; it corresponds to the reciprocal of the bulk modulus. It is also of value to define the concept of acoustic impedance that is the product of the speed of sound by the mass density of the tissue.

\section{Ultrasound wave propagation}

Elastic waves traveling into biological tissues experience different physical phenomena. A few concepts are relevant to explain these wave properties. Reflection, 
Table 1 Glossary of commonly used terms in quantitative ultrasound

\begin{tabular}{|c|c|}
\hline Term & Definition \\
\hline Absorption & Loss in energy of the propagating compression wave due to local tissue heating \\
\hline Acoustic impedance & Product of the speed of sound by the density of the tissue \\
\hline Attenuation & $\begin{array}{l}\text { Decrease in amplitude of acoustic waves propagating through soft tissues; caused by loss of } \\
\text { mechanical energy due to wave absorption, reflection, refraction, diffraction, and scattering }\end{array}$ \\
\hline Attenuation coefficient & $\begin{array}{l}\text { Ratio of one radiofrequency echo magnitude to another at a different depth; expressed in decibel } \\
\text { per centimeter per megahertz }(\mathrm{dB} / \mathrm{cm} / \mathrm{MHz})\end{array}$ \\
\hline Attenuation coefficient slope & Linear relation between the attenuation coefficient and frequency; expressed in $\mathrm{dB} / \mathrm{cm}$ \\
\hline Backscatter & Analysis of echoes received by the transducer due to reflection and scattering of compression waves \\
\hline Backscatter coefficient (BSC) & $\begin{array}{l}\text { Formal definition of the backscatter intensity returned by a tissue and defining its sub-resolution } \\
\text { structure; expressed in } \mathrm{cm} / \text { steradian }\end{array}$ \\
\hline Brightness mode (B-mode) & Ultrasound mode providing two-dimensional images in grayscale for assessment of anatomy \\
\hline Compression wave & $\begin{array}{l}\text { Type of acoustic wave in which the oscillation motion is parallel to the direction of wave propaga- } \\
\text { tion; also known as a longitudinal wave }\end{array}$ \\
\hline Diffraction & $\begin{array}{l}\text { Type of interaction between a wave and a physical medium in which the sound is dispersed when } \\
\text { travelling through a hole smaller than the wavelength }\end{array}$ \\
\hline Homodyned-K (HDK) statistical models & $\begin{array}{l}\text { Descriptive statistical model used to fit the histogram distribution of ultrasound speckle with } 3 \\
\text { parameters for tissue characterization }\end{array}$ \\
\hline In-phase and quadrature (I/Q) demodulated data & $\begin{array}{l}\text { Low-frequency representation of the radiofrequency signal obtained by the quadrature demodula- } \\
\text { tion process }\end{array}$ \\
\hline Nakagami statistical models & $\begin{array}{l}\text { Descriptive statistical model used to fit the histogram distribution of ultrasound speckle with } 2 \\
\text { parameters for tissue characterization }\end{array}$ \\
\hline Point spread function (PSF) & $\begin{array}{l}\text { Response of an ultrasound system to a single reflector much smaller than the acoustic wavelength } \\
\text { but with sufficient impedance to generate an echo }\end{array}$ \\
\hline Quantitative ultrasound (QUS) & $\begin{array}{l}\text { Field of ultrasound imaging that aims to quantify the interactions between a compression acoustic } \\
\text { wave and a biological tissue for its structural sub-resolution characterization }\end{array}$ \\
\hline Radiofrequency (RF) & $\begin{array}{l}\text { Acoustic signals detected by the ultrasound transducer with a frequency bandwidth dictated by the } \\
\text { ultrasound probe characteristics }\end{array}$ \\
\hline Reflection & $\begin{array}{l}\text { Type of interaction between an acoustic wave and a physical medium in which the wave bounces } \\
\text { back at the same angle but at a different direction; reflections generate an echo detected by the } \\
\text { ultrasound transducer when transmitted and reflected angles are in the field of view of the probe }\end{array}$ \\
\hline Refraction & $\begin{array}{l}\text { Type of interaction between an acoustic wave and a physical medium in which the wave is bent at } \\
\text { an angle and travels at a different speed due to a mismatch in acoustic impedance of encountered } \\
\text { tissue interfaces }\end{array}$ \\
\hline Scattering & $\begin{array}{l}\text { Type of interaction between an acoustic wave and a physical medium in which the wave in bounces } \\
\text { at angles of } 360 \text { degrees; scattering occurs when the tissue structure is much smaller than the } \\
\text { acoustic wavelength }\end{array}$ \\
\hline Shear wave & $\begin{array}{l}\text { Type of acoustic wave in which the oscillation motion is perpendicular to the direction of the wave } \\
\text { propagation; also known as transverse waves }\end{array}$ \\
\hline Speed of sound (SoS) & Square root of the bulk elasticity modulus of the tissue divided by its density; expressed in $\mathrm{m} / \mathrm{s}$ \\
\hline Structure factor size estimator (SFSE) & $\begin{array}{l}\text { Spectral representation of the backscatter coefficient modeled with } 2 \text { fitting parameters obtained by } \\
\text { considering wave interference phenomena with a structure factor term, from which is extracted the } \\
\text { packing factor and the mean size of scatterers }\end{array}$ \\
\hline Ultrasound tissue characterization (UTC) & $\begin{array}{l}\text { Historical term to describe the field of quantitative ultrasound; nowadays, the term QUS is preferred } \\
\text { because UTC is also used to describe image processing strategies to extract image characteristics of } \\
\text { a tissue }\end{array}$ \\
\hline
\end{tabular}

refraction, absorption, and scattering phenomena illustrated in Fig. 3, and defining ultrasound image characteristics, are determined by the speed of sound and tissue mass density, yielding the acoustic impedance. More specifically, these wave phenomena are influenced by changes in acoustic impedance along the ultrasound propagation path. All ultrasound imaging modes (Fig. 1) are affected by tissue mechanical properties (bulk or shear modulus), which are hence not specific to ultrasound elastography. Wave diffraction (Fig. 3e) characterized by the spreading of a wave around obstacles or within "holes" created by a mismatch in acoustic impedance is influenced by the relative dimension of the obstacle or hole with respect to the ultrasound wavelength $\lambda$ ( $\lambda$ is the reciprocal of the ultrasound wave frequency). 

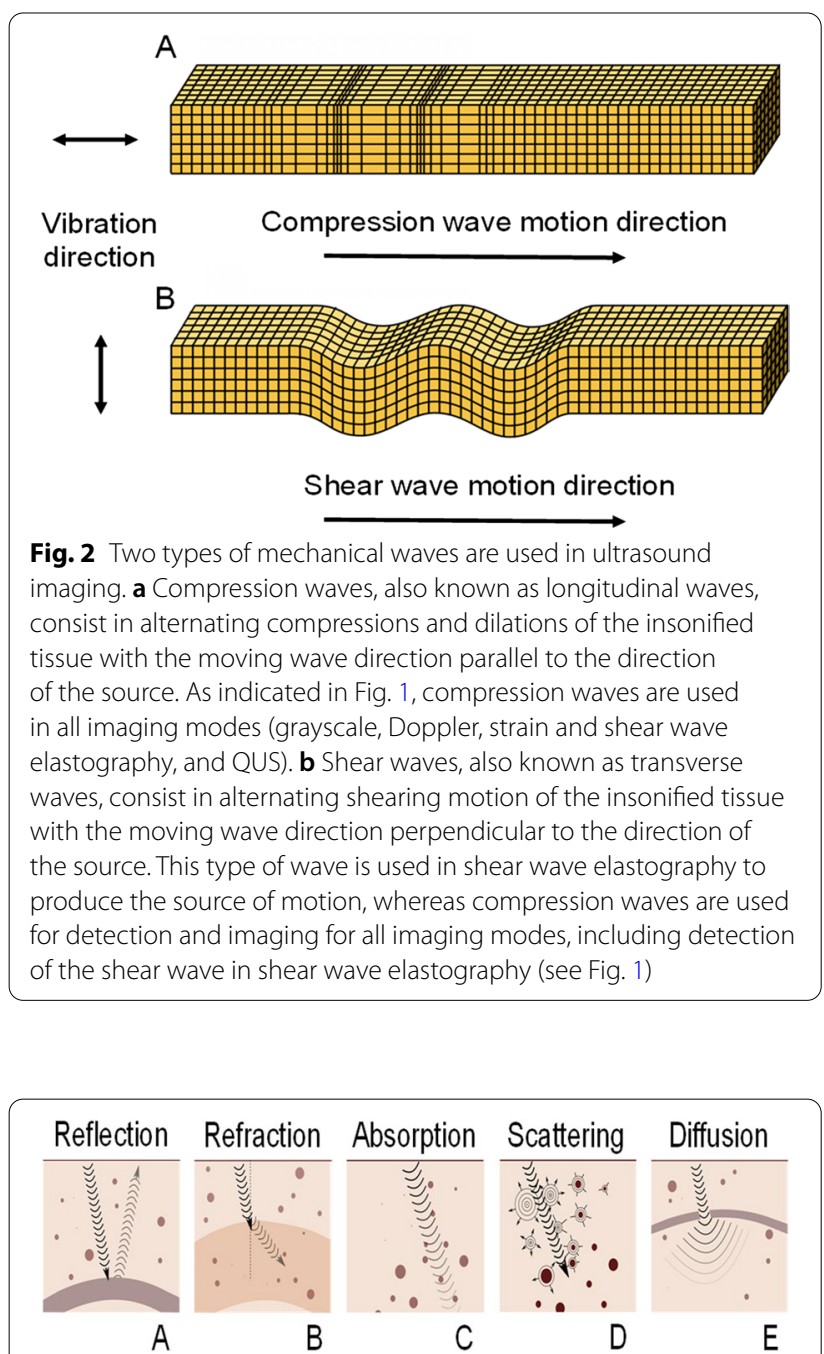

Fig. 3 During their propagation, compression or shear waves are modified due to their interaction with the physical medium. Their direction and amplitude may change due to (a) reflection or (b) refraction at the interface of media with different acoustic impedance. The amplitude may also decrease (i.e., attenuation) due to (c) absorption and (d) scattering produced by the insonified medium. e Space between scatterers or within specular reflectors favor the spreading of the wave field due to diffraction. Waves received by the ultrasound transducer to produce an image are attributed to reflection and scattering

\section{Image formation and point spread function}

As illustrated in Fig. 4, the speckle in B-mode images actually provide a signature of the tissue microstructural cellular content, but at a resolution determined by the system's "point spread function" (PSF), which acts as a "blurring convolution filter." The shape of the PSF is determined by the electrical signal transmitted to probe piezoelectric or capacitive elements, by the beam forming scheme, and by the acoustic lens characteristics. The electrical signal transmitted to the probe

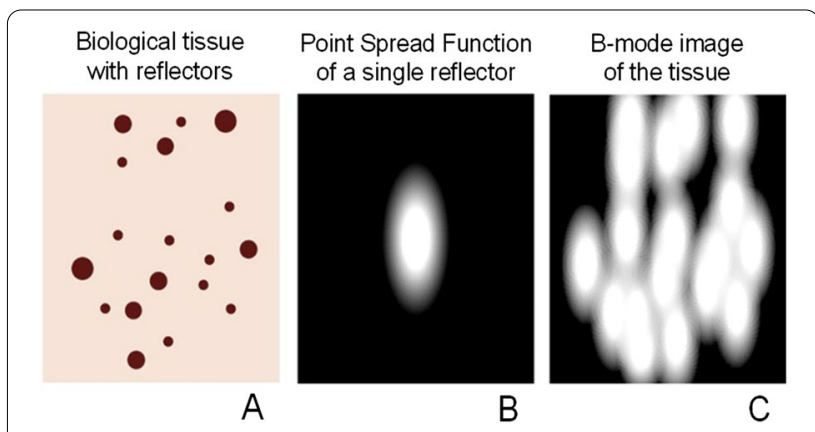

Fig. 4 B-mode images do not provide a resolved signature of the tissue cellular content. a Single cells or structured connected cells forming a living tissue are providing acoustic impedance interfaces reflecting or scattering compressive waves emitted by the ultrasound probe. $\mathbf{b}$ These backward waves are detected by the same probe but are filtered by the "point spread function" of the ultrasound system. In other words, the limited resolution of ultrasound coupled with probe characteristics and scanner settings do not allow resolving single cell geometry. The "point spread function" can be interpreted as the image that would be produced by a single reflector much smaller than the acoustic wavelength but with enough acoustic impedance contrast with the ambient medium to provide a detected backward echo. c The B-mode image corresponds to the tissue sub-resolution characteristics filtered by the "point spread function."The spatial distribution of reflectors and scatterers impact the final image appearance oscillates according to the transducer geometry and fabrication characteristics. The oscillation duration defines the transducer frequency bandwidth and axial resolution (typically $1.5 \mathrm{~mm}$ at $1 \mathrm{MHz}$ ). Its shape (e.g., sinus, square, or wavelet characteristics) modifies the frequency content of the transmitted compression wave, and its dominant frequency defines the mid-bandwidth. Beam forming also affects PSF characteristics, which is used to improve the focus laterally. An acoustic lens defining the focus in elevation (i.e., out of plane of the image) also modulates the PSF behavior and speckle characteristics. Other ultrasound system settings can be used to improve the image quality, and intervene in defining the magnitude and geometry of the PSF [19-21]. An interpretation of the PSF filtering, which characterizes ultrasound systems, is to consider the image that would be produced by a single reflector much smaller than the acoustic wavelength but with enough acoustic impedance contrast to provide a detected backward echo (Fig. 4b). As seen, that reflector cannot be resolved due to this filtering effect. By considering all reflectors depicted in Fig. 4a, the PSF contributes to the speckle pattern observed on B-mode images (this is known as the convolution effect mentioned above, Fig. 4c). The "reflector" terminology used in Figs. 4 and 5 is generic and is referring to reflection or scattered wave phenomena produced by an object. 


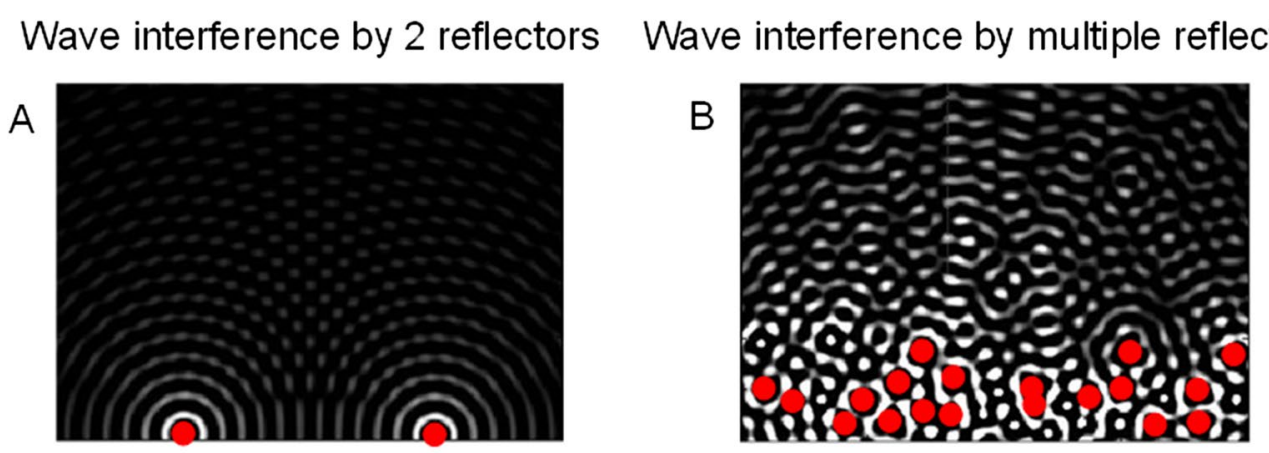

Speckle pattern for different point spread functions

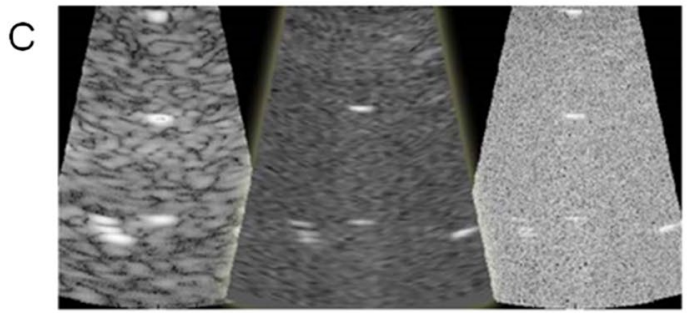

Fig. 5 a Constructive and destructive wave interference patterns when forward echoes sent by a transducer (on the top of the image) are reflected or scattered by 2 particles located at the same depth. $\mathbf{b}$ Wave interference patterns when reflectors or scatterers are distributed in space. $\mathbf{c}$ B-mode images with different speckle patterns obtained from same reflectors or scatterers distributed in space, but with different point spread functions at lower to higher frequencies from left to right. The terminology "reflector" is generic here but formally a reflector has a dimension larger than the acoustic wavelength, whereas a scatterer has a dimension much smaller than the wavelength

\section{Constructive and destructive wave interferences}

When an ultrasound wave interacts with densely packed tissue constituents, constructive and destructive wave interferences are generated: the number and spatial positioning of reflectors are also key determinants of the speckle image characteristics and are a source of the sub-resolution information gathered by QUS backscatter imaging biomarkers. The previous explanation on image formation is oversimplified because a given reflector does not lead to a specific speckle defined by the PSF (as in the case of Fig. 4c). When multiple reflectors are present (Fig. 5a, b, we consider here weak scatterers - i.e., small acoustic impedence contrasts - thus multiple reflections between scatterers are negligable), wave interferences are observed and this is also contributing to the speckle pattern characteristics. The PSF and wave interferences are modulating image characteristics. As illustrated in Fig. 5c, a given tissue with specific positioning of reflectors that is imaged with different scanners or system settings leading to different PSFs would result in quite different B-mode image appearance. The concept of constructive and destructive wave interferences is further illustrated in Fig. 6. If one considers two reflectors producing echoes with different delays among them due to different spatial positioning, the summation of these waves contributing to the final image pixel can be quite different according to the importance and value of this delay. If two echoes are in phase (i.e., no delay between them), then a constructive interference with a doubled

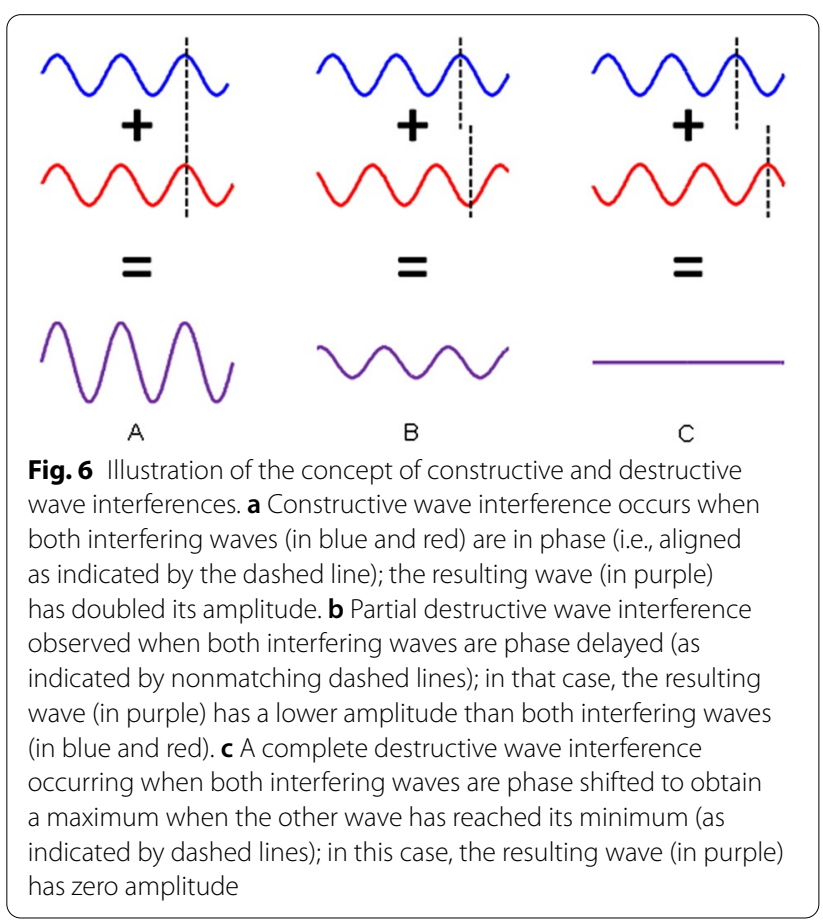


final amplitude is observed. If two echoes are out of phase (i.e., with a phase shift of 180 degrees), then the wave interference will produce a null echo. This is typical of black pixels observed on B-mode images when an underlying tissue is expected. Intermediate conditions with phase delays between 0 and 180 degrees lead to different levels of wave interferences.

Advanced materials: Intuitively, one may think that more reflectors or scatterers present in a given ROI would produce brighter images (or higher backscatter in the context of QUS), but this is not true because of wave interferences. Experimental reports using flowing red blood cells or cellulose particles embedded in a gel phantom at different volume concentrations revealed a linear relation between the backscatter intensity and the number of particles up to a volume fraction of approximately $6 \%$, followed by a peak around 13-20\% volume fractions, and a reduction in backscatter at higher number densities of particles (i.e., hematocrit in the case of blood) [22, 23]. Destructive wave interferences explain the reduction of the echo magnitude as the number of particles is increased beyond typically 13-20\% volume fractions. This observation might be of value to interpret blood backscatter in anemia, or liver backscatter at different lipid vesicular concentrations in steatosis [24].

$\mathrm{RF}$ mode at the origin of all ultrasound imaging modalities All imaging modes in ultrasound start from radiofrequency (RF) data processing. RF images correspond to the summation of echoes received by each element of a transducer following beam forming to improve image resolution. As indicated in Fig. 1, imaging modes are specifically processing RF data, in-phase and quadrature (I/Q) demodulated data, or simply B-mode images, as in the case of speckle tracking for cardiac strain imaging [25]. Following beam forming, a B-mode image is obtained from side-by-side RF lines (usually with interpolation) that are processed to obtain I/Q and then envelop detected echoes (i.e., a B-mode echo). The Doppler mode is based on the processing of the demodulated I/Q data to retrieve the phase shift between emitted and received echoes to apply the Doppler equation for assessing blood velocities [26]. In strain or shear wave elastography, RF data processing is privileged to improve tissue movement detection accuracy, but I/Q or B-mode data processing can be used for some algorithms [27]. I/Q data are advantageous to reduce sampling rate and data transfer load, whereas B-mode processing is of value because of the wide access to clinical B-mode image sequences.

Notice that 15-20 years ago, a few ultrasound manufacturers started providing access to RF data for research purpose (e.g., Ultrasonix Medical Corporation, Verasonics, Visualsonics, Esaote, Terason). Today, most major clinical ultrasound manufacturers can provide RF or I/Q access in addition to clinical imaging packages (e.g., Siemens Healthineers, General Electrics Healthcare, Canon Medical Systems, Samsung Healthcare, Supersonic Imagine-Hologic, etc.). Research agreements may, however, still be required to have RF access with some companies. Acquiring RF data had a major impact because it provided academic scientists the possibility of developing technical innovations with state-of-the-art ultrasound scanners.

\section{$\mathrm{RF}, \mathrm{I} / \mathrm{Q}$ and $\mathrm{B}$-mode data formats}

Figure 7 illustrates the difference between RF, I/Q, and B-mode datasets (adapted from [28]). The left panel shows time-domain processing, and the right panel presents the corresponding spectral content. The timedomain RF signal contains detected reflected and scattered echoes returning to the transducer. It is understood that constructive and destructive wave phenomena contribute to the RF signal signature. Since transmitted compression waves are selected to provide frequency contents covering the whole bandwidth of the transducer (typically by using a short transient electrical signal), and considering that frequency characteristics are modified by frequency-dependent phenomena, such as attenuation and backscatter, the received compression waves can be seen as a "filtered" representation of transmitted echoes. Once detected by the transducer in analogic form (i.e., as a time-varying voltage), analog-to-digital converters are used to obtain a numerical representation for further processing. As indicated in the top right panel of Fig. 7, the frequency content of the RF signal is in the $\mathrm{MHz}$ range and corresponds to the transducer bandwidth (e.g., 1.25 to $3.75 \mathrm{MHz}$ for this example that might correspond to an abdominal or cardiac probe). Time-domain processing of the RF signal provides the low-frequency I/Q representation of received echoes with corresponding spectral description on the right panel. Following envelope detection (red line on the left panel) made by processing time-domain or frequency-domain echoes, its magnitude is mapped in gray levels to represent B-mode speckle at a given lateral (i.e., a given RF scan line) and depth (i.e., a given time) positions to produce an image.

\section{Introduction to QUS imaging}

Figure 8 provides an intuitive representation of speed of sound, attenuation, and backscatter measurements. Most algorithms and methods developed to provide these QUS biomarkers are based on RF data processing. RF data are more suited to track wave motion required for speed of sound imaging, and they contain spectral information used to describe attenuation and backscatter measures as a function of frequency. Since QUS imaging provides 


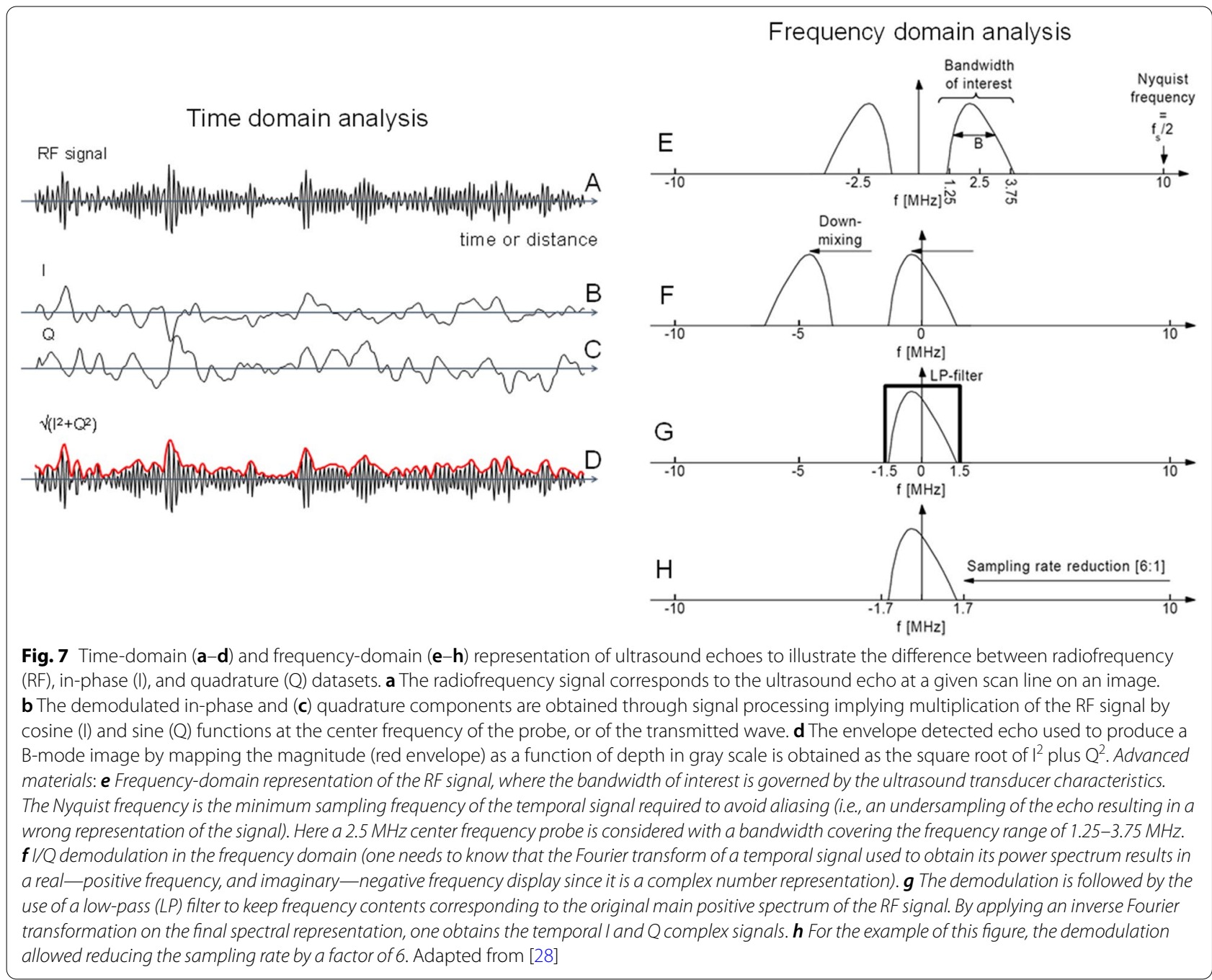

sub-resolution information relying on the cellular content and structure of a tissue, it could also be angular dependent when the measure is done on anisotropic tissues, such as muscles or tendons having fibers organized with privileged orientations. Examples of parametric images of these biomarkers are given in specific sections describing how these imaging modes are obtained.

\section{Speed of sound imaging \\ Concept}

Biological tissues are characterized by different speed of sound $(\mathrm{SoS})$ providing acoustic impedance contrast.

\section{Units}

SoS is expressed in meters per second $(\mathrm{m} / \mathrm{s})$.

\section{Range}

Reported values measured using experimental ultrasound devices and excised soft tissues are typically varying from 1400 to $1700 \mathrm{~m} / \mathrm{s}$ at body temperature [1]. SoS is lower in fatty tissues and higher in muscles and tendons, and it changes according to the pathological state of the tissue [29]. Most reports on SoS are based on technical experimental setups; tabulated values obtained using clinical implementation of the methods are scarce.

\section{Measurement methods}

Original efforts in this field aimed at improving the quality of B-mode images corrupted by phase aberrations produced by varying $\mathrm{SoS}$ along the acoustic wave propagation path [30-33]. As discussed earlier (in advanced materials), a heterogeneous tissue with varying bulk moduli and mass densities can locally modify the SoS, and consequently the depth at which each component of the tissue would be represented. For example, the boundary of an organ with spatially varying SoS might be displayed at different depths due to compression wave phase aberrations, potentially 


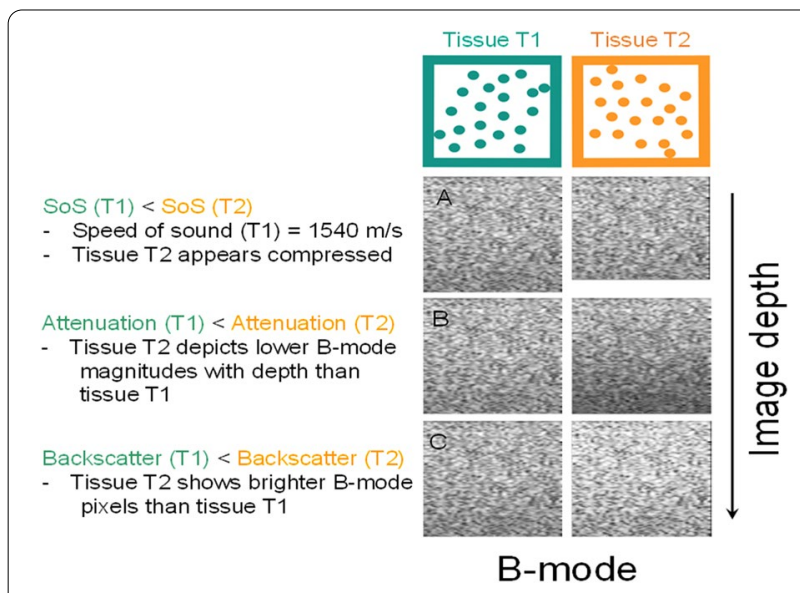

Fig. 8 Simple interpretation of QUS imaging modes. Tissues T1 and $\mathrm{T} 2$ when interacting with a compression wave emitted by the transducer are characterized by different QUS properties evaluated by the speed of sound (SoS), attenuation, and backscatter coefficient measures. The interpretation of QUS parameters in this example is based on the B-mode representation of the tissue. a Compared with a reference tissue $T 1$ that has a speed of sound of $1540 \mathrm{~m} / \mathrm{s}$, tissue $T 2$ that has a higher speed of sound would appear compressed because distances on clinical ultrasound systems are measured with the assumption that all tissues behave with an SoS $=1540 \mathrm{~m} / \mathrm{s}$. b Tissue $\mathrm{T} 2$ that has a higher attenuation would appear hypoechoic in deeper locations. c Tissue T2 that has a higher backscatter would appear hyperechoic on B-mode imaging

affecting the quality of the diagnosis. The objective of phase aberration correction methods is to adjust locally the SoS to produce clearer images with less blurring. Technical efforts made in the field of phase aberration corrections constitute the framework of contemporary SoS measurement and imaging methods in the QUS field.

Advanced materials: Phase aberration correction methods led to the development of focusing strategies relying on the estimation of the mean SoS between the face of the transducer and the focal depth of interest. Alternatively, an image processing approach also aiming at improving beam focusing was proposed [34]. In the latter report, the mathematical deconvolution operator was used to retrieve a restored image produced by considering an ultrasound system PSF having different mean SoS values. A focusing approach based on the estimation of SoS along the wave propagation path was specifically introduced for the purpose of providing a tissue characterization signature in [35]. Conceptually, since a mean estimate along the ultrasound beam is obtained with these abovementioned methods [34, 35], no local measure within a given ROI of an organ having inhomogeneous SoS can be produced. Current methods allowing local assessments or images within an ROI are based on spatial coherence [36] and image compounding [37] approaches.

\section{Illustration}

A schematic representation of the current state-of-theart spatial coherence [36] and image compounding [37] methods developed for local SoS assessments is illustrated in Fig. 9. Another recent strategy was proposed for producing local SoS maps based on a prior estimate of the mean SoS along the wave propagation path [38].

\section{Clinical example(s)}

A few ultrasound manufacturers are today offering SoS capability for tissue characterization by providing a mean value within a selected ROI (no image), as in [39]. Imaging local heterogeneous SoS pixel values within an ROI might become available soon to clinicians; an example of such capability in the context of breast cancer imaging is given in [37], see Fig. 10 (image compounding method). It is anticipated that multiple organ diagnosis based on SoS images might spread once manufacturers will release such imaging packages. SoS measurement methods and instruments were also proposed in the context of QUS imaging using tomographic reconstructions [40-43].

\section{Attenuation imaging Concept}

Ultrasound attenuation refers to the loss of mechanical energy as an acoustic wave propagates in soft tissues [4]. In addition to compression wave absorption (i.e., transfer of energy into heat), wave reflection, refraction, diffraction, and scattering are also contributing to the diminution of the wave amplitude with distance by redirecting acoustic energy away from the field of view of the transducer. The attenuation can be qualitatively appreciated by clinicians performing a standard B-mode examination. The magnitude of the echo within the image is reduced by attenuation, and loss of structural details over depth and shadowing can be observed. Thus, attenuation may be considered as an imaging artifact or as a specific feature of the tissue with diagnostic value. For a proper understanding of concepts described below, "total" attenuation means the attenuation between the ultrasound probe and an organ at a given depth (thus considering the contribution of all tissues along the path, e.g., the skin, fat layers, muscles, blood vessels, etc.), whereas "local" attenuation refers to the attenuation within an organ ROI to provide a tissue signature. Images might be reported using both total and local attenuation measurements. The characterization of an organ based on QUS attenuation measures or images usually refers to local attenuation assessment. 


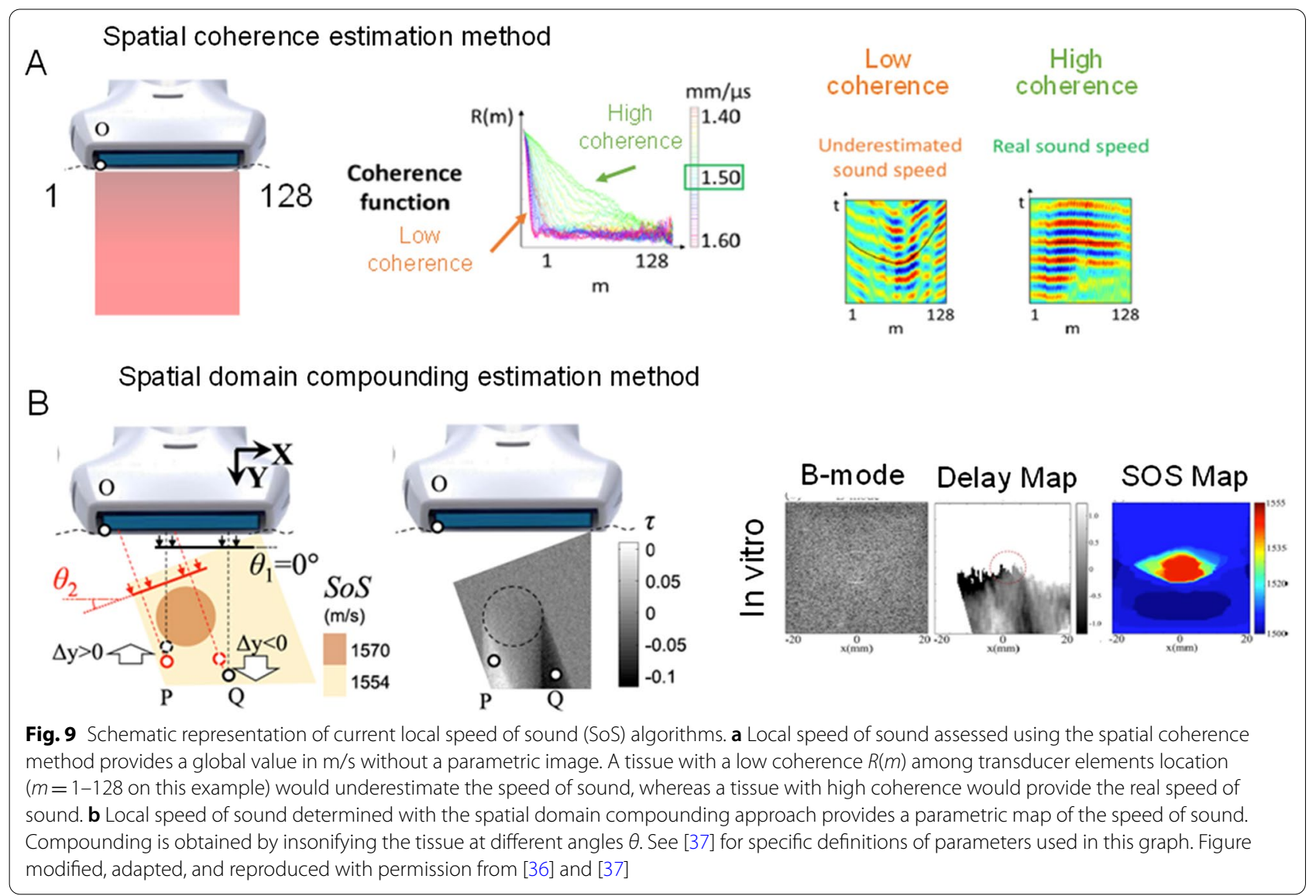

\section{Units}

Since attenuation is the ratio of one RF magnitude to another at a different depth, it is expressed using the unit of decibel $(\mathrm{dB})$ computed as 20 times the logarithm (in base 10) of this ratio. Also, because ultrasound

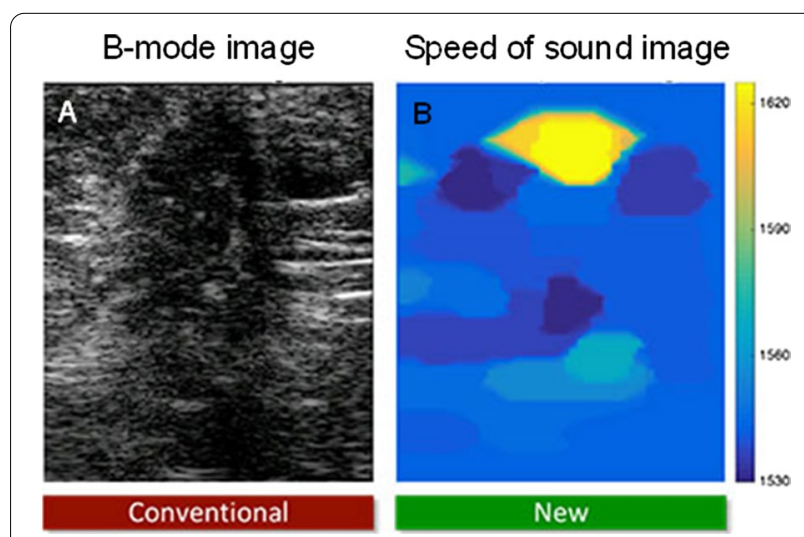

Fig. 10 a B-mode image of an 88-year-old woman with a breast cancer. $\mathbf{b}$ Speed of sound map computed within the selected region of interest considered for B-mode imaging. Figure provided by Orcun Goksel from the Swiss Federal Institute of Technology, Zurich, Switzerland. Related works can be found in [37] attenuation increases with frequency, it is reported in $\mathrm{dB} / \mathrm{cm} / \mathrm{MHz}$. If one assumes that the attenuation on a log scale varies linearly with frequency (which is not the case of all biological tissues [44]), the value at a higher frequency may be obtained by multiplying the attenuation in $\mathrm{dB} / \mathrm{cm} / \mathrm{MHz}$ by the frequency in $\mathrm{MHz}$ to obtain a measure in $\mathrm{dB} / \mathrm{cm}$ at a given frequency (which is often reported). By assuming such a linear dependency with frequency, the relation between attenuation versus frequency is obtained by computing the slope of this relation; for this reason, it is common to report attenuation measures with the acronym "attenuation coefficient slope" (ACS).

\section{Range}

Experimental measures of the ultrasound attenuation coefficient at body temperature using through transmission (i.e., 2 transducers located on both sides of the tissue) or reflection (i.e., one transducer for emission and reception) instrumentations vary from $0.01 \mathrm{~dB} / \mathrm{cm} / \mathrm{MHz}$ in blood to $4 \mathrm{~dB} / \mathrm{cm} / \mathrm{MHz}$ in muscles $[1,45]$. These values correspond to extremes reported in technical reports based on experimental setups and ex vivo tissue samples. 


\section{Measurement methods}

There are two main methods for estimating the local attenuation coefficient slope, i.e., the attenuation in $\mathrm{dB} /$ $\mathrm{cm} / \mathrm{MHz}$ within a pre-specified ROI: the spectral difference $[46,47]$ and the spectral shift [48] methods. With the former approach, the attenuation coefficient slope is estimated from the reduction of the echo signal power with depth, whereas for the latter, it is deduced from the downshift in center frequency of the backscatter echo with depth due to the frequency-dependent attenuation. Variants of these strategies were proposed, namely the spectral log difference method [49] and a hybrid method [50]. These algorithms are assuming that the scattering properties (i.e., the backscatter coefficient) of the organ are unchanged over the depth range of the ROI. Moreover, a calibration method is required to compensate for the compression wave diffraction confounder of the transducer, which also reduces the echo magnitude with depth. As illustrated in Fig. 11, a compression wave emitted by an ultrasound transducer does not have uniform magnitude laterally and axially following beam forming; consequently, a calibration is required to compensate for spatial changes in magnitude.

To perform the calibration, echo signals from a reference phantom whose attenuation is known must be obtained using the same equipment and system settings as the clinical examination. Such calibration phantoms are available commercially (e.g., Sun Nuclear or CIRS). Because of the often assumed linear frequency dependence of the logarithm representation of local attenuation, such calibration phantoms may be used for the whole frequency bandwidth of clinical array transducers. The ratio of power spectra from the tissue and reference phantom within an ROI yields the attenuation coefficient of the scanned organ at the frequency and depth of interest. System effects such as diffraction, beam forming, and transmitted acoustic power are accounted for by applying the reference phantom method [51]. Tissue diffraction (Fig. 3e) is a QUS signature captured by attenuation measurements. Gain, time-gain compensation, and image filtering settings on clinical scanners, which affect B-mode images, usually do not impact RF images and thus the computation of attenuation.

\section{Illustration}

A schematic illustration of the contemporary spectral difference and spectral shift methods used to assess local attenuation is given in Fig. 12. It displays an ROI within a B-mode image with measurement windows used for computation, along with a simplified representation of the phantom calibration approach.

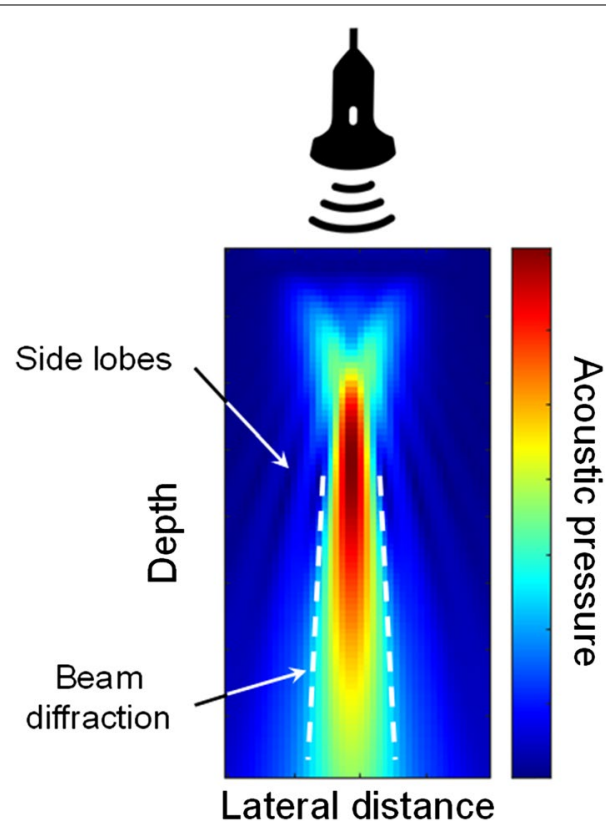

Fig. 11 Typical diffraction pattern of an ultrasound probe and presence of side lobes associated with the beam forming reconstruction method, and finite dimension of the transducer aperture (typically determined by the number of transducer elements used at emission and reception). Notice that diffraction and side lobe characteristics are defining the point spread function property schematized in Fig. 4. This example illustrates the acoustic pressure distribution of a typical ultrasound beam produced by an array transducer. Specific beam forming strategies are used to reduce side lobes and multiple focuses improve the uniformity of the acoustic pressure distribution. Nevertheless, wave diffraction occurs and should be compensated to achieve reliable QUS measurements. Because the magnitude of compression waves is not uniform in the lateral ( $x$-axis) and axial ( $y$-axis or depth) directions, calibration with a reference phantom is required to compensate for spatial changes

\section{Clinical example(s)}

A few ultrasound manufacturers are offering real-time attenuation images on their scanners [52-55], or values computed within an ROI with no imaging capability [56-58]. Other manufacturers reported post-processed RF datasets on remote computers with no capability of real-time attenuation imaging [59-62]. To compensate for ultrasound system wave diffraction, ultrasound beam focusing, and other system settings, most manufacturers opted for an embedded calibration (from either a training dataset or a preset reference phantom measurement). Figure 13 shows an example of local attenuation imaging implemented on a commercial clinical system for the assessment of liver steatosis.

\section{Backscatter imaging Concept}

Backscatter imaging refers to the analysis of echoes received by the ultrasound transducer due to 

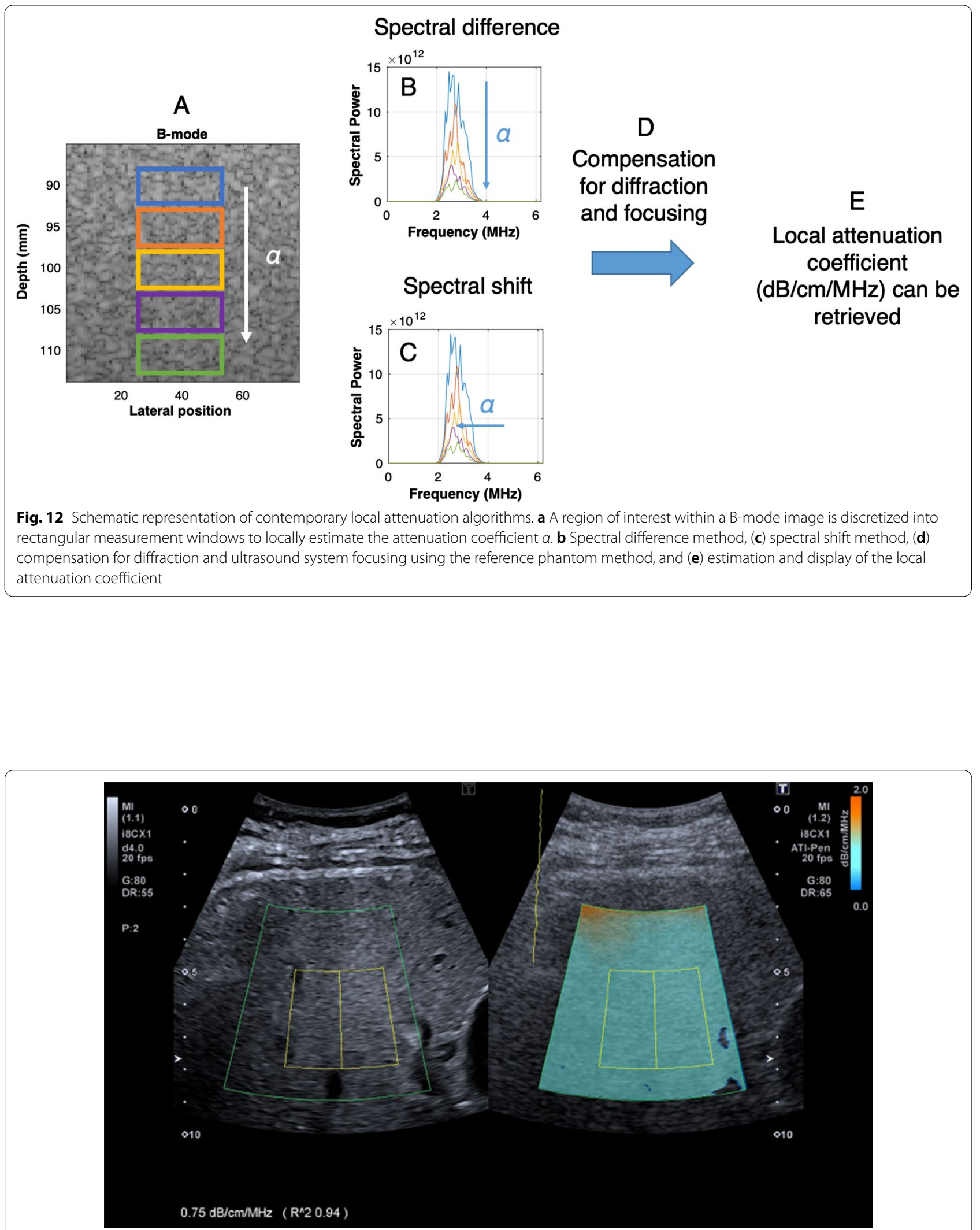

Fig. 13 Local attenuation map of a 55-year-old man with nonalcoholic fatty liver disease reporting a mean attenuation within the selected ROI of $0.75 \mathrm{~dB} / \mathrm{cm} / \mathrm{MHz}$, indicating a stage 1 to 3 liver steatosis. Reproduced with permission from [54] 
compression wave reflection and scattering, which are modulated in magnitude by constructive and destructive wave interferences produced by the tissue microstructure. A backscatter image therefore reflects the tissue microstructure, but varies with ultrasound frequency. For assessing the backscatter tissue property, a compensation for compression wave attenuation along the propagation path should be considered to get a signature of the intrinsic distribution in acoustic impedance inhomogenities of the tissue producing backscatter echoes. This is accomplished by considering the total attenuation along the path between the emitted compression wave at the surface of the skin to the ROI within the insonified organ $[63,64]$.

\section{Units}

The most recognized approach to describe backscatter in the field of QUS imaging is to use the magnitude squared and frequency dependency of RF echoes received by the transducer to compute the backscatter coefficient (BSC) [65]. By definition, the BSC at a given frequency corresponds to the time-averaged scattered intensity in the backward direction per unit solid angle per unit volume normalized by the time-averaged incident wave intensity $\left(\mathrm{cm}^{-1} \mathrm{Sr}^{-1}\right)$ [4]. Practical implementation may require sampling at different probe positions and/or orientations to get spatial averaging of the tissue BSC. Units are per $\mathrm{cm}$ and per steradian, where the latter is a dimensionless measure referring to the ratio of the area subtended by the square of its distance from the center to that distance squared, as illustrated in Fig. 14. In this figure, the origin

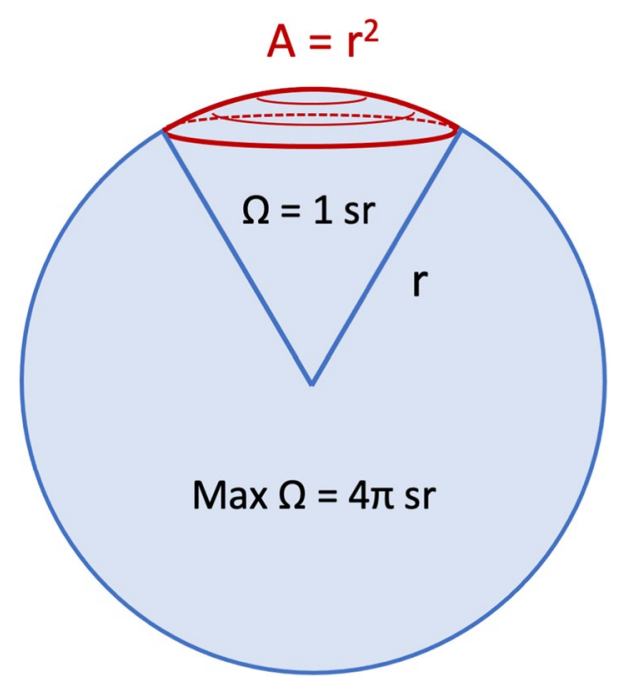

Fig. 14 Illustration of the steradian dimensionless unit used to define the backscatter coefficient. The solid three-dimensional angle $\Omega$ corresponds to the area $A$ divided by the radius squared of the sphere of the subtended angle may correspond to the central position of a scatterer within a specific ROI comprising multiple acoustic scatterers.

Advanced materials: By definition, BSC $<1$ (no backscatter amplification) unless hypothetically a specific ROI would contain scatterers with a reflection coefficient of 1 (i.e., no loss in energy between transmitted and reflected waves, which would correspond to a huge contrast in acoustic impedance), and these scatterers would need to be organized spatially in such a way that mainly constructive wave interferences would be present. Such conditions are not expected for biological soft tissues so that BSC is a fractional measure.

\section{Range}

As for previous SoS and local attenuation values, BSC measurements have been experimental and mainly based on laboratory instruments; approximate extreme values are given here for soft biological tissues. Reported values for porcine whole blood at a normal hematocrit and a frequency of $7.5 \mathrm{MHz}$ vary from $\approx 0.1$ to $5 \times 10^{-3} \mathrm{~cm}^{-1}$ $\mathrm{Sr}^{-1}$. The range of BSCs for blood at a given frequency is explained by the modulating effect of erythrocyte aggregation and flow condition [22,66]. The mean BSC at $3 \mathrm{MHz}$ of human fatty livers is $\approx 7 \times 10^{-3} \mathrm{~cm}^{-1} \mathrm{Sr}^{-1}$ compared with a mean value of $\approx 0.5 \times 10^{-3} \mathrm{~cm}^{-1}$ $\mathrm{Sr}^{-1}$ in healthy livers [67]. At low frequencies (typically $<10 \mathrm{MHz}$ ), BSC has a frequency dependency varying from $\approx f^{2}$ to $f^{4}$ so that values at higher frequency are much higher for a given tissue. When the frequency is further increased, oscillating behavior with BSC peaks and troughs is observed and corresponds typically to the microstructure of the tissue and mean scatterers' size.

\section{Measurement methods}

Similarly to local attenuation estimation requiring a reference phantom calibration method, backscatter assessment using the BSC also needs a calibration to account for ultrasound system characteristics and settings [68]. A requirement for BSC measurements is to obtain a reference BSC in $\mathrm{cm}^{-1} \mathrm{Sr}^{-1}$ at a specific frequency, or at different frequencies within the bandwidth of the ultrasound transducer, to compare the RF signal from the insonified organ to that of the reference for calibration. Commercially available reference phantoms provide BSC values but usually at a single frequency. Contrary to local attenuation in $\mathrm{dB} / \mathrm{cm}$ that varies almost linearly with frequency, the BSC has a more complex tissue specific frequency behavior $[3,4]$, as mentioned above. Consequently, additional effort is often required to calibrate the $\mathrm{BSC}$ of the reference phantom at a specific frequency or frequencies of interest. 
As summarized by Wear et al. [65], the assessment of the BSC has been associated with a greater variability than SoS or attenuation measurements due to the greater variety of measurement procedures, algorithms, and mathematical formulation relying on specific scattering theories. Using a more standardized way to compute the BSC with clinical array transducers, more robust estimates have been reported in vitro $[69,70]$ and in vivo $[71$, 72]. The framework of all BSC methods relies on similar concepts [73].

\section{Illustration}

Figure 15 shows requirements necessary to report calibrated and robust BSC values; measurements are typically taken in the frequency domain using Fourier transforms applied to RF signals. With a reference phantom properly calibrated to provide known BSCs within the bandwidth of the transducer, a clinical measurement of RF datasets within a specified ROI (i.e., tissue T1) at a given depth in an organ is taken, followed by a second measurement on the reference phantom for the same ROI, keeping all ultrasound system settings the same. As indicated in Fig. 15, the mean RF power spectrum of the clinical measures computed over a few frames is divided by the mean RF power spectrum of the reference phantom, and then the result is multiplied by a function used to compensate for total attenuation. For this purpose, the total attenuation of the reference phantom at the measurement window depth should be known, along with an estimate of the total attenuation at the measurement window location within the imaged organ. The latter measure might be quite challenging, especially in obese patients with layered fat structures above the organ of interest [70]. For this reason, recent

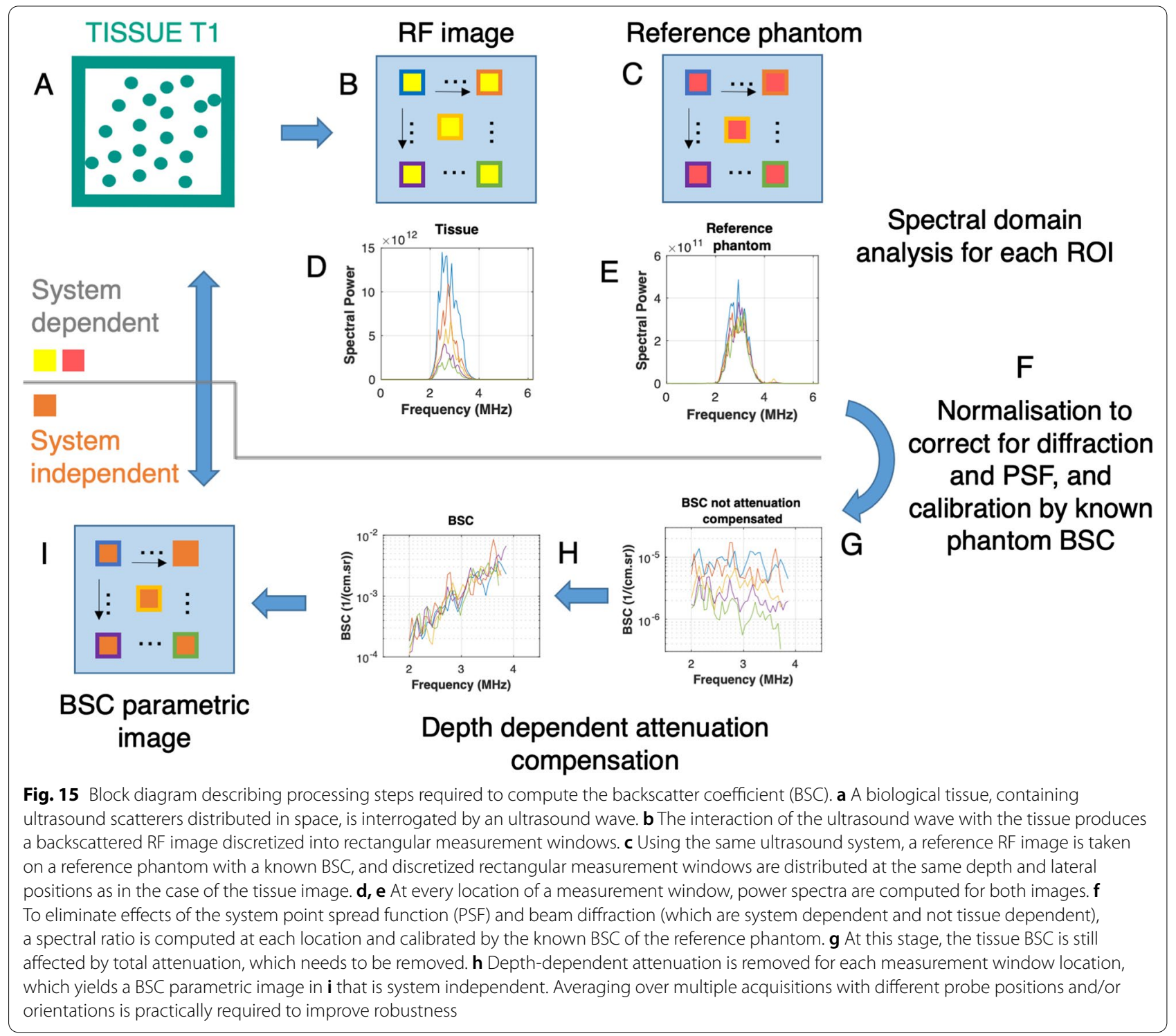


clinical reports assumed a constant total attenuation coefficient slope between the probe and the liver ROI for steatosis grading characterization with the BSC [74, 75]. As indicated in Fig. 15, because the BSC is computed within measurement windows of a given ROI (i.e., tissue T1) in an organ, methods used either the total attenuation up to the measurement window or the total attenuation up to the ROI added to the local attenuation up to the measurement window. One assumption in the calculation of the BSC is the postulate of a similar speed of sound for the clinical tissue sample and reference phantom, which can be a source of variability.

Advanced materials: As mentioned above, a first prerequisite for BSC computation is the availability of a reference phantom with a known BSC for the calibration of diffraction and ultrasound system settings. If such phantom is not available or not calibrated at the frequency of interest or within the frequency bandwidth considered, a pre-step additional calibration is required. This is done by using another reference phantom mimicking specific theoretical scattering conditions. Notice that a few fundamental research laboratories may have the expertise to fabricate such phantoms, and to match acquired RF spectra to the appropriate theoretical scattering model. Once the pre-calibration phantom is fabricated, it is usually recommended to calibrate the reference commercial phantom for clinical use. Indeed, the lifetime of such pre-calibration phantoms is usually limited by dehydration, bacterial contamination or structural fragility, thus preventing them to be used as a reference for clinical BSC measurements.

The theoretical Faran model describing scattering by hard spheres is the most often used for BSC calibration [76]; it is appropriate to model the BSC of a low number density of small glass spheres randomly distributed within a solidified gel, which is a common fabrication process for BSC reference phantoms. The requirement of a low number density of scatterers is to avoid constructive and destructive wave interferences not supported by this theoretical model. It also avoids multiple reflections due to the use of glass spheres with a high acoustic impedance mismatch with the surrounding gel. With proper total attenuation compensation, RF spectra acquired on the pre-calibration phantom embedding scatterers of a uniform size are then compared to the Faran model to calibrate the commercially available reference phantom at frequencies of interest. This is achieved by repeating measurements on that commercial phantom whilst keeping ultrasound system settings the same.

Alternatively for blood BSC assessment on patients to assess specific conditions, such as systemic inflammation [77] or risk of deep vein thrombosis [78], a suspension of erythrocytes washed with saline to remove plasma proteins that are responsible for cell aggregation is a common practice for reference phantom calibration [79]. To avoid wave interference effects, a low 4-6\% hematocrit suspension datasets are used and compared to the Percus-Yevick scattering model $[80,81]$ to obtain the reference BSC(f). An advantage of this calibration process is to keep similar flow condition between calibration and clinical measurements [82], since it is affecting the BSC of blood.

\section{Clinical example(s)}

To our knowledge, no ultrasound manufacturer has yet implemented quantitative BSC on their scanners. However, technology releases are in development [62] and are using an embedded calibration with preset reference phantom measurements. Alternatively, relative backscatter measures (or relative echogenicity) have widely been reported, as in the case of the hepatorenal index implemented on a few clinical scanners for liver steatosis characterization [83]. However, such measures depend on the instrument, organ assessed or used for normalization (e.g., kidney in the case of the hepatorenal index, or blood and artery wall adventitia in the case of atherosclerotic plaque analysis [84]).

\section{Investigational backscatter imaging methods}

Advanced materials: Modeling approaches were proposed to consider the whole bandwidth of the BSC instead of a single measurement at a given frequency. The rationale is relevant because the spectral representation of the BSC and especially its oscillating frequency behavior may provide unique description of the sub-resolution structural characterization of a tissue. Numerous reports thus described backscatter descriptive approaches to fit the behavior of $B S C(f)$ to retrieve imaging biomarkers [4]. We do not aim at being exhaustive but simple metrics such as the spectral slope, mid-band fit, and spectral intercept have been widely used. For example, these metrics, which do not rely on physical modeling but on frequency fitting characteristics of $B S C(f)$, allowed following breast cancer treatment response to chemotherapy [85]. By considering a Gaussian-shaped form factor of scatterers distributed randomly within an insonified tissue, physical modeling of $B S C(f)$ allowed retrieving other diagnostic features, known as the effective scatterer diameter and effective acoustic concentration [86]. Images based on these backscatter metrics have been reported for in vivo tissue analyses [87]. Inspired by blood backscatter modeling, for which a structure factor was introduced to model constructive and destructive wave interferences attributed to the scatterers' spatial positioning [88], BSC physical models considering a form factor of scatterers, and a structure factor, were proposed and used for spectral analyses of other 
tissues than blood [89-91]. The model behind these latter developments is known as the structure factor size estimator (SFSE) [92].

\section{Statistical backscatter modeling}

Alternatively to the BSC, first-order statistical properties of the RF echo envelope (i.e., B-mode speckle) have been used as a signature of the tissue microstructure [93]. Pioneer works showed potential of these backscatter measures for breast and liver image analyses [94, 95]. The basic concept relies on modeling the magnitude of speckle with probability density functions. Figure 16 illustrates the concept of speckle analysis with homodyned-K (HDK) and Nakagami statistical models. Parameters used to fit the model to a histogram distribution representing the dataset can be presented in the form of color parametric images overlaid on B-mode images for diagnosis [96].

Recently, an ultrasound manufacturer has released backscatter imaging based on Nakagami modeling [55, 97] (Fig. 17). The color map in Fig. 17 may indicate that proprietary post-processing was performed because the range does not correspond to expected values for the scale and shape parameters of this model. This is a technique that has advantages in terms of reduction of the computational complexity when compared with the BSC,

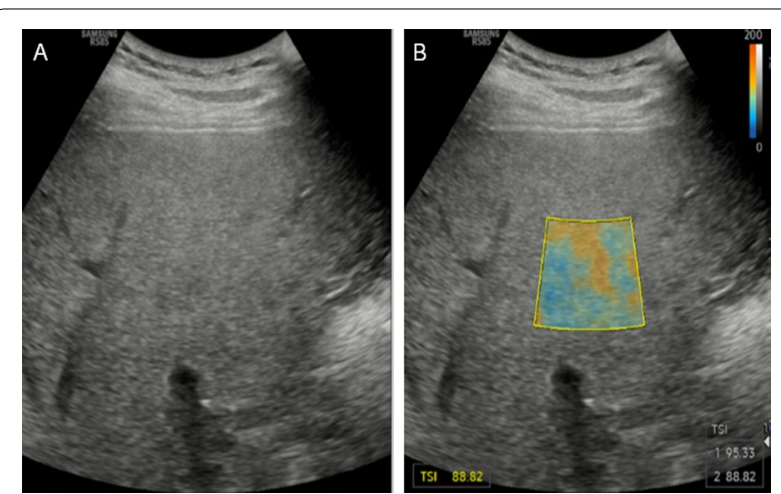

Fig. 17 a B-mode image of an unidentified patient with suspected liver steatosis. b Tissue scattering imaging (TSI) based on Nakagami histogram modeling and post-processing. Reproduced with permission from [55]

but the independence to ultrasound system settings and total attenuation remains to be fully validated. Alternatively, another ultrasound manufacturer is proposing an imaging mode based on normalized variance statistics [98].

Advanced materials: Before concluding this educational review, a last explanation is given to help appreciating

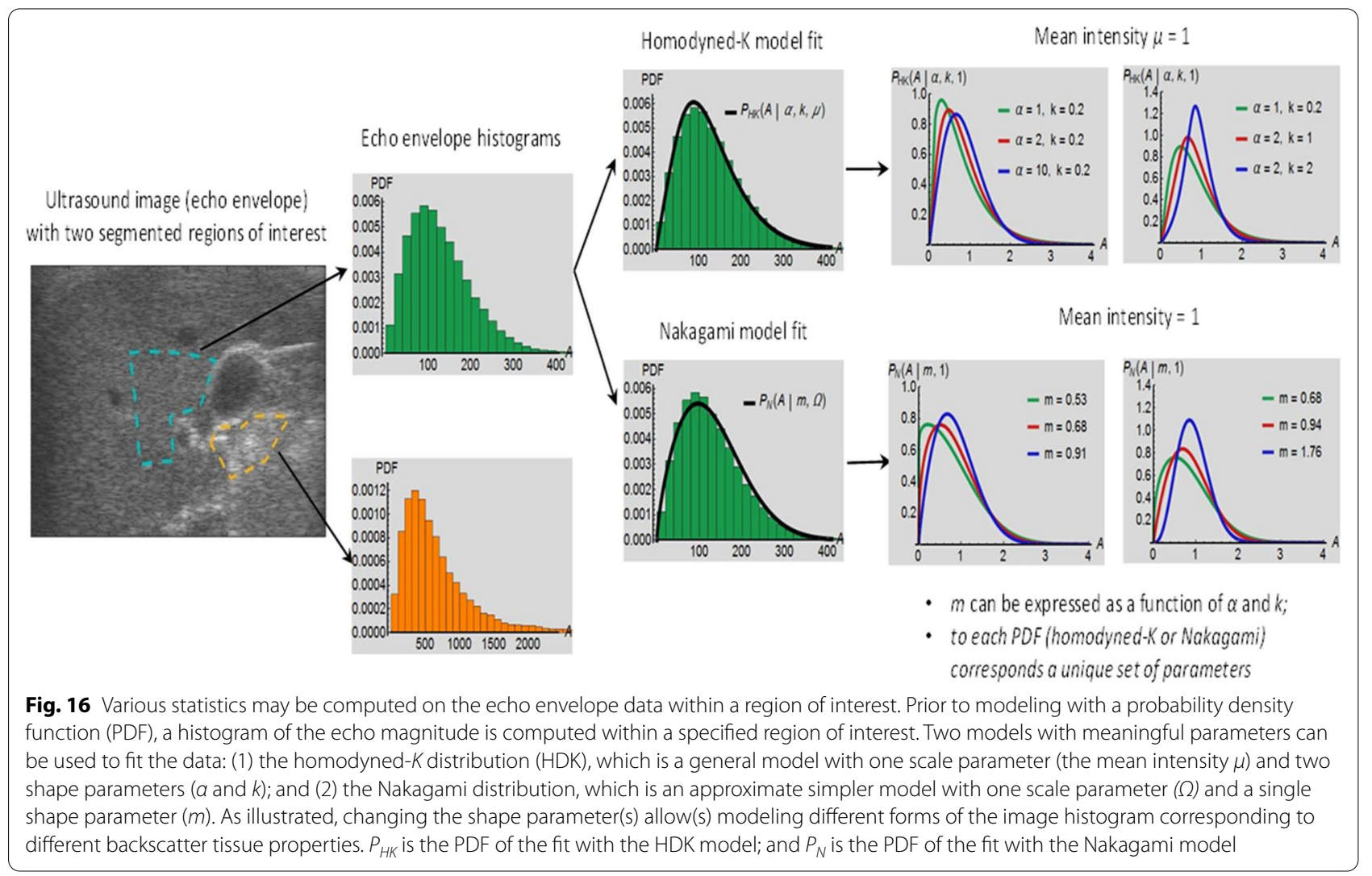


the relevance of HDK or Nakagami tissue modeling for QUS imaging. Long standing developments in the field of QUS aimed at interpreting or providing structural meaning to fitted parameters obtained from statistical speckle models. This was mainly done through acoustic physics simulations or comparison of model parameters with ex vivo tissue histopathology slides. Without going into details, unifying concepts were recently made between BSC spectral modeling with the SFSE, and HDK statistical representation of backscatter echoes [99, 100]. The total, coherent (i.e., from spatially organized scatterers), and diffuse (i.e., from randomly distributed scatterers in space) signal powers related to HDK modeling were expressed explicitly in terms of the structure factor of the BSC SFSE model [99]. Also, the scatterer clustering parameter of the HDK model ( $\alpha$ in Fig. 16) could be related to the packing factor of the SFSE model. We can thus conclude that $B S C(f)$ and HDK speckle modeling (or Nakagami modeling) share common sub-resolution tissue descriptors.

\section{Future directions}

The field of QUS imaging is very active and recent innovations have aimed at improving image quality by using mathematical regularization algorithms [49, 101-106]. Indeed, most QUS imaging methods rely on computations made over windows that are typically larger than the PSF (5-10 times), which degrades the spatial resolution compared to B-mode imaging. Spatial filtering might be employed to reduce the window effect but regularization has the additional advantage of reducing computed outlier values appearing as background noise on QUS images. The development of phantom-free methods in QUS imaging is also a trend to pursue to improve the clinical workflow [107]. The efforts made by a few ultrasound manufacturers in this direction deserve to be acknowledged.

Artificial intelligence has started to be introduced in the field of QUS imaging to improve the tissue sub-resolution signature [108]; this may certainly play a major role in the future. Notice finally that the assessment of the total attenuation between the ultrasound probe and the tissue of interest remains a challenge for improving the robustness of BSC computation [64]. Mathematical optimization algorithms were proposed to simultaneously model the frequency dependency of the BSC with an assessment of the total attenuation [109-111]. Robustness and validation of these methods remain to be demonstrated.

A last word goes to the QIBA-PEQUS (quantitative imaging biomarkers alliance-pulse echo quantitative ultrasound) committee of the Radiological Society of North America and American Institute of Ultrasound in Medicine that should release soon guidelines on the clinical use of QUS imaging methods for liver steatosis assessment. A similar effort is conducted by the World Federation of Ultrasound in Medicine and Biology [112].

\section{Conclusion}

QUS has been investigated for more than 50 years and is undergoing a resurgence, particularly for liver steatosis applications. The recent introduction of dedicated instruments using QUS techniques and implementation of speed of sound, local attenuation, and backscatter statistical packages on clinical scanners should contribute to their clinical adoption. Backscatter coefficient packages may also become available soon on clinical scanners. This educational review emphasized the fact that the ultrasound image texture is determined by the relationship between the spatial distribution of acoustic scatterers, their acoustic impedance discrepancy with respect to surrounding tissues, and characteristics of the ultrasound source. Acoustic scatterers in biological tissues are neither randomly nor perfectly ordered, and are generally present at a high number density, which has an impact on wave propagation. The ultrasound image texture is determined by constructive and destructive wave interferences affected by the density of scatterers, and their distribution in space. QUS methods provide a signature of tissues' physical property, which may be represented as parametric images for diagnosis. The introduction of QUS techniques in clinical care should provide additional diagnostic tools to clinicians.

\section{Abbreviations}

ACS: Attenuation coefficient slope; BSC: Backscatter coefficient; HDK: Homodyned-K; I/Q: In-phase and quadrature datasets; PDF: Probability density function; PSF: Point spread function; QUS: Quantitative ultrasound; RF: Radiofrequency; ROI: Region of interest; SFSE: Structure factor size estimator; SoS: Speed of sound; UTC: Ultrasound tissue characterization.

\section{Acknowledgements \\ Authors acknowledge the contribution of Dr Pol Grasland-Mongrain who provided the framework of some figures.}

\section{Authors' contributions}

GC wrote the manuscript; FD and FY conceptualized some figures; GC, FD, FY, and AT reviewed and improved the structure of the manuscript; GC, FY, and AT provided funding contributing to innovations in the field of quantitative ultrasound imaging. All authors read and approved the final manuscript.

\section{Funding}

Funding for this educational work was partially supported by: Canadian Institutes of Health Research (\#84358 to GC; \#389385 to AT and GC); Natural Sciences and Engineering Research Council of Canada (\#CHRP-508337 and \#503381 to GC; \#RGPIN-2018-04233 to FY); Canadian Cancer Research Society (\#19075 to GC); OncoTech (joint funding from Fonds de recherche Québec, Oncopole, Medteq, TransMedTech, Canadian Cancer Research Society, and Siemens Healthineers to GC and AT); Fonds de recherche du Québec en Santé (FRQ-S \#254072 Junior 1 Salary Award to FY); and Fonds de recherche du Québec en Santé and Fondation de l'association des radiologistes du Québec 
(FRQS-ARQ \#34939 Clinical Research Scholarship - Junior 2 Salary Award to AT).

\section{Availability of data and materials}

No data or material is available for public distribution.

\section{Declarations}

\section{Ethics approval and consent to participate}

This educational review contains no data or figures attributed to clinical protocols conducted at the University of Montreal Hospital Research Center. Examples of clinical results were taken from the literature under approval to reproduce materials.

\section{Consent for publication}

All authors contributed to the content of this article and consent for publication.

\section{Competing interests}

Authors GC and AT have research agreements with Siemens Healthcare Limited for projects on liver steatosis and liver cancer using quantitative ultrasound technologies.

\section{Author details}

${ }^{1}$ Laboratory of Biorheology and Medical Ultrasonics, Centre de recherche du Centre hospitalier de I'Université de Montréal (CRCHUM), 900 St-Denis, Montréal, Québec H2X 0A9, Canada. ${ }^{2}$ Department of Radiology, Radio-oncology, and Nuclear Medicine, Université de Montréal, Montréal, Québec, Canada. ${ }^{3}$ Institute of Biomedical Engineering, Université de Montréal, Montréal, Québec, Canada. ${ }^{4}$ Microbubble Theranostics Laboratory, CRCHUM, Montréal, Québec, Canada. ${ }^{5}$ Department of Radiology, Centre hospitalier de I'Université de Montréal (CHUM), Montréal, Québec, Canada. ${ }^{6}$ Laboratory of Medical Image Analysis, Montréal, CRCHUM, Canada.

Received: 18 June 2021 Accepted: 7 August 2021

Published online: 09 September 2021

\section{References}

1. Greenleaf JF (1986) Tissue characterization with ultrasound I, 1st edn. CRC Press Inc, Boca Raton

2. Greenleaf JF (1986) Tissue characterization with ultrasound II, 1st edn. CRC Press, Boca Raton

3. Shung KK, Thieme GA (1993) Ultrasonic Scattering in Biological Tissues, 1st edn. CRC Press, Boca Raton

4. Mamou J, Oelze ML (2013) Quantitative ultrasound in soft tissues, 1st edn. Springer, Netherlands

5. Madsen EL, Dong F, Frank GR et al (1999) Interlaboratory comparison of ultrasonic backscatter, attenuation, and speed measurements. J Ultrasound Med 18:615-631

6. Laugier P, Haïat G (2011) Bone quantitative ultrasound. Springer

7. Sasso M, Miette V, Sandrin L, Beaugrand M (2012) The controlled attenuation parameter (CAP): a novel tool for the non-invasive evaluation of steatosis using Fibroscan. Clin Res Hepatol Gastroenterol 36:13-20

8. Linzer M (1976) The ultrasonic tissue characterization seminar: an assessment. J Clin Ultrasound 4:97-100

9. Gluer CC (1997) Quantitative ultrasound techniques for the assessment of osteoporosis: expert agreement on current status. The International Quantitative Ultrasound Consensus Group. J Bone Miner Res 12:1280-1288

10. Njeh CF, Boivin CM, Langton CM (1997) The role of ultrasound in the assessment of osteoporosis: a review. Osteoporos Int 7:7-22

11. Zhou Z, Wu W, Wu S, Jia K, Tsui PH (2017) A review of ultrasound tissue characterization with mean Scatterer spacing. Ultrason Imaging 39:263-282

12. Docking SI, CookJ (2016) Pathological tendons maintain sufficient aligned fibrillar structure on ultrasound tissue characterization (UTC). Scand J Med Sci Sports 26:675-683
13. Landau LD, Lifshitz EM (2002) Theory of elasticity, 3rd edn. Butterworth Heinemann, Oxford

14. Morse PM, Ingard KU (1986) Theoretical acoustics. Princeton University Press, New Jersey

15. Nenadic IZ, Urban MW, Aristizabal S, Mitchell SA, Humphrey TC, Greenleaf JF (2011) On Lamb and Rayleigh wave convergence in viscoelastic tissues. Phys Med Biol 56:6723-6738

16. Shih CC, Qian X, Ma T et al (2018) Quantitative assessment of thin-layer tissue viscoelastic properties using ultrasonic micro-elastography with lamb wave model. IEEE Trans Med Imaging 37:1887-1898

17. Goss SA, Johnston RL, Dunn F (1980) Compilation of empirical ultrasonic properties of mammalian tissues. II. J Acoust Soc Am 68:93-108

18. Anderson ME, McKeag MS, Trahey GE (2000) The impact of sound speed errors on medical ultrasound imaging. J Acoust Soc Am 107:3540-3548

19. Cobbold RSC (1974) Transducers for biomedical measurements: principles and applications, 1st edn. Wiley, New York

20. Christensen DA (1988) Ultrasonic bioinstrumentation, 1st edn. Wiley, New York

21. McDicken WN (1991) Diagnostic ultrasonics: principles and use of instruments, 3rd edn. Churchill Livingstone, Edinburgh

22. Yuan YW, Shung KK (1988) Ultrasonic backscatter from flowing whole blood. I: Dependence on shear rate and hematocrit. J Acoust Soc Am 84:52-58

23. Chen JF, Zagzebski A, Madsen EL (1996) Experimental demonstration of the frequency dependence of the effective scatterer number density. J Acoust Soc Am 99:1932-1936

24. Gesnik M, Bhatt M, Roy Cardinal MH et al (2020) In vivo ultrafast quantitative ultrasound and shear wave elastography imaging on farm-raised duck livers during force feeding. Ultrasound Med Biol 46:1715-1726

25. Geyer H, Caracciolo G, Abe H et al (2010) Assessment of myocardial mechanics using speckle tracking echocardiography: fundamentals and clinical applications. J Am Soc Echocardiogr 23:351-369

26. Evans DH, McDicken WN (2000) Doppler ultrasound: physics, instrumentation and signal processing, 2nd edn. Wiley, Chichester

27. Nenadic IZ, Urban MW, Greenleaf JF, Gennisson JL, Bernal M, Tanter M (2019) Ultrasound elastography for biomedical applications and medicine. Wiley, New York

28. Kirkhorn J (1999) Introduction to IQ-demodulation of RF-data. Norwegian University of Science and Technology, Trondheim, pp 1-13

29. Bamber JC, Hill CR (1981) Acoustic properties of normal and cancerous human liver-I. Dependence on pathological condition. Ultrasound Med Biol 7:121-133

30. Flax SW, O'Donnell M (1988) Phase-aberration correction using signals from point reflectors and diffuse scatterers: basic principles. IEEE Trans Ultrason Ferroelectr Freq Control 35:758-767

31. Nock L, Trahey GE, Smith SW (1989) Phase aberration correction in medical ultrasound using speckle brightness as a quality factor. J Acoust Soc Am 85:1819-1833

32. Napolitano D, Chou CH, McLaughlin G et al (2006) Sound speed correction in ultrasound imaging. Ultrasonics 44(Suppl 1):e43-46

33. Cho MH, Kang LH, Kim JS, Lee SY (2009) An efficient sound speed estimation method to enhance image resolution in ultrasound imaging. Ultrasonics 49:774-778

34. Shin HC, Prager R, Gomersall H, Kingsbury N, Treece G, Gee A (2010) Estimation of average speed of sound using deconvolution of medical ultrasound data. Ultrasound Med Biol 36:623-636

35. Hayashi N, Tamaki N, Senda M et al (1988) A new method of measuring in vivo sound speed in the reflection mode. J Clin Ultrasound 16:87-93

36. Imbault M, Faccinetto A, Osmanski BF et al (2017) Robust sound speed estimation for ultrasound-based hepatic steatosis assessment. Phys Med Biol 62:3582-3598

37. Sanabria SJ, Ozkan E, Rominger M, Goksel O (2018) Spatial domain reconstruction for imaging speed-of-sound with pulse-echo ultrasound: simulation and in vivo study. Phys Med Biol 63:215015

38. Jakovljevic M, Hsieh S, Ali R, Chau Loo Kung G, Hyun D, Dahl JJ (2018) Local speed of sound estimation in tissue using pulse-echo ultrasound: model-based approach. J Acoust Soc Am 144:254-266 
39. Popa A, Sirli R, Popescu A et al (2021) Ultrasound-based quantification of fibrosis and steatosis with a new software considering transient elastography as reference in patients with chronic liver diseases. Ultrasound Med Biol 47:1692-1703

40. Andre MP, Wiskin J, Borup D (2013) Clinical results with ultrasound computed tomography of the breast. In: Mamou J, Oelze ML (eds) Quantitative ultrasound in soft tissues. Springer, Dordrecht, pp 395-432

41. Li C, Duric N, Littrup P, Huang L (2009) In vivo breast sound-speed imaging with ultrasound tomography. Ultrasound Med Biol 35:1615-1628

42. Jirik R, Peterlik I, Ruiter N et al (2012) Sound-speed image reconstruction in sparse-aperture 3-D ultrasound transmission tomography. IEEE Trans Ultrason Ferroelectr Freq Control 59:254-264

43. Khodr ZG, Sak MA, Pfeiffer RM et al (2015) Determinants of the reliability of ultrasound tomography sound speed estimates as a surrogate for volumetric breast density. Med Phys 42:5671-5678

44. D'Astous FT, Foster FS (1986) Frequency dependence of ultrasound attenuation and backscatter in breast tissue. Ultrasound Med Biol 12:795-808

45. Nassiri DK, Nicholas D, Hill CR (1979) Attenuation of ultrasound in skeletal muscle. Ultrasonics 17:230-232

46. Insana M, Zagzebski J, Madsen E (1983) Improvements in the spectral difference method for measuring ultrasonic attenuation. Ultrason Imaging 5:331-345

47. Parker KJ, Waag RC (1983) Measurement of ultrasonic attenuation within regions selected from B-scan images. IEEE Trans Biomed Eng BME-30:431-437

48. Samimi K, Varghese T (2015) Performance evaluation of the spectral centroid downshift method for attenuation estimation. IEEE Trans Ultrason Ferroelectr Freq Control 62:871-880

49. Coila AL, Lavarello R (2018) Regularized spectral log difference technique for ultrasonic attenuation imaging. IEEE Trans Ultrason Ferroelectr Freq Control 65:378-389

50. Kim H, Varghese T (2008) Hybrid spectral domain method for attenuation slope estimation. Ultrasound Med Biol 34:1808-1819

51. Rosado-Mendez IM, Nam K, Hall TJ, Zagzebski JA (2013) Task-oriented comparison of power spectral density estimation methods for quantifying acoustic attenuation in diagnostic ultrasound using a reference phantom method. Ultrason Imaging 35:214-234

52. Ferraioli G, Maiocchi L, Raciti MV et al (2019) Detection of liver steatosis with a novel ultrasound-based technique: a pilot study using MRIderived proton density fat fraction as the gold standard. Clin Transl Gastroenterol 10:e00081

53. Bae JS, Lee DH, Lee JY et al (2019) Assessment of hepatic steatosis by using attenuation imaging: a quantitative, easy-to-perform ultrasound technique. Eur Radiol 29:6499-6507

54. Ferraioli G, Maiocchi L, Savietto G et al (2021) Performance of the attenuation imaging technology in the detection of liver steatosis. J Ultrasound Med 40:1325-1332

55. Jeon SK, Lee JM, Joo I (2021) Clinical feasibility of quantitative ultrasound imaging for suspected hepatic steatosis: intra- and inter-examiner reliability and correlation with controlled attenuation parameter. Ultrasound Med Biol 47:438-445

56. Tamaki N, Koizumi Y, Hirooka M et al (2018) Novel quantitative assessment system of liver steatosis using a newly developed attenuation measurement method. Hepatol Res 48:821-828

57. Cerit M, Sendur HN, Cindil E et al (2020) Quantification of liver fat content with ultrasonographic attenuation measurement function: correlation with unenhanced multidimensional computerized tomography. Clin Imaging 65:85-93

58. Koizumi Y, Hirooka M, Tamaki N et al (2019) New diagnostic technique to evaluate hepatic steatosis using the attenuation coefficient on ultrasound B mode. PLoS One 14:e0221548

59. Fujiwara $Y$, Kuroda $H$, Abe $T$ et al (2018) The B-mode image-guided ultrasound attenuation parameter accurately detects hepatic steatosis in chronic liver disease. Ultrasound Med Biol 44:2223-2232

60. Tada T, lijima H, Kobayashi $\mathrm{N}$ et al (2019) Usefulness of attenuation imaging with an ultrasound scanner for the evaluation of hepatic steatosis. Ultrasound Med Biol 45:2679-2687
61. Tada T, Kumada T, Toyoda $\mathrm{H}$ et al (2020) Liver stiffness does not affect ultrasound-guided attenuation coefficient measurement in the evaluation of hepatic steatosis. Hepatol Res 50:190-198

62. Labyed Y, Milkowski A (2020) Novel method for ultrasound-derived fat fraction using an integrated phantom. J Ultrasound Med 39:2427-2438

63. Bigelow TA, Labyed $Y$ (2013) Attenuation compensation and estimation. In: Mamou J, Oelze ML (eds) Quantitative ultrasound in soft tissues. Springer, New York, pp 71-93

64. Coila A, Rouyer J, Zenteno O, Luchies A, Oelze ML, Lavarello R (2021) Total attenuation compensation for backscatter coefficient estimation using full angular spatial compounding. Ultrasonics 114:106376

65. Wear KA, Stiles TA, Frank GR et al (2005) Interlaboratory comparison of ultrasonic backscatter coefficient measurements from 2 to $9 \mathrm{MHz}$. J Ultrasound Med 24:1235-1250

66. Shung KK, Yuan YW, Fei DY, Tarbell JM (1984) Effect of flow disturbance on ultrasonic backscatter from blood. J Acoust Soc Am 75:1265-1272

67. Lu ZF, Zagzebski JA, Lee FT (1999) Ultrasound backscatter and attenuation in human liver with diffuse disease. Ultrasound Med Biol 25:1047-1054

68. Yao LX, Zagzebski JA, Madsen EL (1990) Backscatter coefficient measurements using a reference phantom to extract depth-dependent instrumentation factors. Ultrason Imaging 12:58-70

69. Nam K, Rosado-Mendez IM, Wirtzfeld LA et al (2012) Cross-imaging system comparison of backscatter coefficient estimates from a tissuemimicking material. J Acoust Soc Am 132:1319-1324

70. Nam K, Rosado-Mendez IM, Wirtzfeld LA et al (2012) Comparison of ultrasound attenuation and backscatter estimates in layered tissuemimicking phantoms among three clinical scanners. Ultrason Imaging 34:209-221

71. Han A, Andre MP, Deiranieh L et al (2018) Repeatability and reproducibility of the ultrasonic attenuation coefficient and backscatter coefficient measured in the right lobe of the liver in adults with known or suspected nonalcoholic fatty liver disease. J Ultrasound Med 37:1913-1927

72. Han A, Zhang YN, Boehringer AS et al (2019) Inter-platform reproducibility of ultrasonic attenuation and backscatter coefficients in assessing NAFLD. Eur Radiol 29:4699-4708

73. Ghoshal G, Mamou J, Oelze ML (2013) State of the art methods for estimating backscatter coefficients. In: Mamou J, Oelze ML (eds) Quantitative ultrasound in soft tissues. Springer, New York, pp 3-19

74. Han A, Zhang YN, Boehringer AS et al (2020) Assessment of hepatic steatosis in nonalcoholic fatty liver disease by using quantitative US Radiology 295:106-113

75. Lin SC, Heba E, Wolfson T et al (2015) Noninvasive diagnosis of nonalcoholic fatty liver disease and quantification of liver fat using a new quantitative ultrasound technique. Clin Gastroenterol Hepatol 13:1337-1345

76. Faran JJJ (1951) Sound scattering by solid cylinders and spheres. J Acoust Soc Am 23:405-418

77. Gyawali P, Ziegler D, Cailhier JF, Denault A, Cloutier G (2018) Quantitative measurement of erythrocyte aggregation as a systemic inflammatory marker by ultrasound imaging: a systematic review. Ultrasound Med Biol 44:1303-1317

78. Yu FTH, Armstrong J, Tripette J, Meiselman HJ, Cloutier G (2011) A local increase in red blood cell aggregation can trigger deep vein thrombosis: evidence based on quantitative cellular ultrasound imaging. J Thromb Haemost 9:481-488

79. Wang SH, Shung KK (1997) An approach for measuring ultrasonic backscattering from biological tissues with focussed transducers. IEEE Trans Biomed Eng 44:549-554

80. Twersky V (1987) Low-frequency scattering by correlated distributions of randomly oriented particles. J Acoust Soc Am 81:1609-1618

81. Lucas RJ, Twersky V (1987) Inversion of ultrasonic scattering data for red blood cell suspensions under different flow conditions. J Acoust Soc Am 82:794-799

82. Chayer B, Allard L, Qin Z et al (2020) Pilot clinical study of quantitative ultrasound spectroscopy measurements of erythrocyte aggregation within superficial veins. Clin Hemorheol Microcirc 74:109-126 
83. Webb M, Yeshua H, Zelber-Sagi S et al (2009) Diagnostic value of a computerized hepatorenal index for sonographic quantification of liver steatosis. AJR Am J Roentgenol 192:909-914

84. Cloutier G, Roy Cardinal MH, Ju Y, Giroux MF, Lanthier S, Soulez G (2018) Carotid plaque vulnerability assessment using ultrasound elastography and echogenicity analysis. AJR Am J Roentgenol 211:847-855

85. Sadeghi-Naini A, Papanicolau N, Falou O et al (2013) Quantitative ultrasound evaluation of tumor cell death response in locally advanced breast cancer patients receiving chemotherapy. Clin Cancer Res 19:2163-2174

86. Lizzi FL, King DL, Rorke MC et al (1988) Comparison of theoretical scattering results and ultrasonic data from clinical liver examinations. Ultrasound Med Biol 14:377-385

87. Oelze ML, Mamou J (2016) Review of quantitative ultrasound: envelope statistics and backscatter coefficient imaging and contributions to diagnostic ultrasound. IEEE Trans Ultrason Ferroelectr Freq Control 63:336-351

88. Yu F, Cloutier G (2007) Experimental ultrasound characterization of red blood cell aggregation using the structure factor size estimator. J Acoust Soc Am 122:645-656

89. Han A, O'Brien W Jr (2015) Structure function for high-concentration biophantoms of polydisperse scatterer sizes. IEEE Trans Ultrason Ferroelectr Freq Control 62:303-318

90. Saha RK, Kolios MC (2011) Effects of cell spatial organization and size distribution on ultrasound backscattering. IEEE Trans Ultrason Ferroelectr Freq Control 58:2118-2131

91. Franceschini E, Guillermin R, Tourniaire F, Roffino S, Lamy E, Landrier JF (2014) Structure factor model for understanding the measured backscatter coefficients from concentrated cell pellet biophantoms. J Acoust Soc Am 135:3620-3631

92. Yu FTH, Franceschini E, Chayer B, Armstrong J, Meiselman HJ, Cloutier G (2009) Ultrasonic parametric imaging of erythrocyte aggregation using the structure factor size estimator. Biorheology 46:343-363

93. Destrempes F, Cloutier G (2013) Review of envelope statistics models for quantitative ultrasound imaging and tissue characterization. In: Mamou J, Oelze ML (eds) Quantitative ultrasound in soft tissues. Springer, New York, pp 1-55

94. Molthen RC, Shankar PM, Reid JM et al (1998) Comparisons of the Rayleigh and k-distribution models using in vivo breast and liver tissue. Ultrasound Med Biol 24:93-100

95. Shankar PM, Dumane VA, George T et al (2003) Classification of breast masses in ultrasonic B scans using Nakagami and K distributions. Phys Med Biol 48:2229-2240

96. Tsui PH, Chang CC (2007) Imaging local scatterer concentrations by the Nakagami statistical model. Ultrasound Med Biol 33:608-619

97. Jeon SK, Joo I, Kim SY et al (2021) Quantitative ultrasound radiofrequency data analysis for the assessment of hepatic steatosis using the controlled attenuation parameter as a reference standard. Ultrasonography 40:136-146

98. Bae JS, Lee DH, Lee JY et al (2020) Quantitative assessment of fatty liver using ultrasound with normalized local variance technique. Ultraschall Med. https://doi.org/10.1055/a-1143-3091
99. Destrempes F, Franceschini E, Yu FT, Cloutier G (2016) Unifying concepts of statistical and spectral quantitative ultrasound techniques. IEEE Trans Med Imaging 35:488-500

100. Destrempes F, Cloutier G (2020) Interpretation based on stochastic geometry of homodyned-K distribution scatterer clustering parameter for quantitative ultrasound imaging. In: IEEE international ultrasonics symposium, pp 1-4

101. Jafarpisheh N, Hall TJ, Rivaz H, Rosado-Mendez IM (2021) Analytic global regularized backscatter quantitative ultrasound. IEEE Trans Ultrason Ferroelectr Freq Control 68:1605-1617

102. Vajihi Z, Rosado-Mendez IM, Hall TJ, Rivaz H (2018) Low variance estimation of backscatter quantitative ultrasound parameters using dynamic programming. IEEE Trans Ultrason Ferroelectr Freq Control 65:2042-2053

103. Deeba F, Schneider C, Mohammed S et al (2019) SWTV-ACE: spatially weighted regularization based attenuation coefficient estimation method for hepatic steatosis detection. In: International conference on medical image computing and computer-assisted intervention, pp 610-618

104. Deeba F, Rohling R (2020) Repeatability and reproducibility of quantitative ultrasound parameter estimation using spatially weighted total variation (SWTV) regularization. In: IEEE ultrasonics symposium, pp 1-4

105. Destrempes F, Gesnik M, Cloutier G (2019) Construction of adaptively regularized parametric maps for quantitative ultrasound imaging. In: IEEE ultrasonics symposium, pp 2027-2033

106. Rafati I, Destrempes F, Cloutier G (2020) Regularized phantom-free construction of local attenuation coefficient slope maps for quantitative ultrasound imaging. In: IEEE ultrasonics symposium, pp 1-4

107. Gong P, Song P, Huang C, Trzasko J, Chen S (2019) System-independent ultrasound attenuation coefficient estimation using spectra normalization. IEEE Trans Ultrason Ferroelectr Freq Control 66:867-875

108. Tehrani AKZ, Amiri M, Rosado-Mendez IM, Hall TJ, Rivaz H (2021) Ultrasound scatterer density classification using convolutional neural networks and patch statistics. IEEE Trans Ultrason Ferroelectr Freq Control 68:2697-2706

109. Franceschini E, Yu FTH, Cloutier G (2008) Simultaneous estimation of attenuation and structure parameters of aggregated red blood cells from backscatter measurements. J Acoust Soc Am 123:EL85-EL91

110. Franceschini E, Yu FTH, Destrempes F, Cloutier G (2010) Ultrasound characterization of red blood cell aggregation with intervening attenuating tissue-mimicking phantoms. J Acoust Soc Am 127:1104-1115

111. Nam K, Zagzebski JA, Hall TJ (2011) Simultaneous backscatter and attenuation estimation using a least squares method with constraints. Ultrasound Med Biol 37:2096-2104

112. Ferraioli G, Berzigotti A, Barr RG et al (2021) Quantification of liver fat content with ultrasound: A WFUMBposition paper. Ultrasound Med Biol. https://doi.org/10.1016/j.ultrasmedbio.2021.06.002

\section{Publisher's Note}

Springer Nature remains neutral with regard to jurisdictional claims in published maps and institutional affiliations.

\section{Submit your manuscript to a SpringerOpen ${ }^{\odot}$ journal and benefit from:}

- Convenient online submission

- Rigorous peer review

- Open access: articles freely available online

- High visibility within the field

Retaining the copyright to your article

Submit your next manuscript at $\boldsymbol{\nabla}$ springeropen.com 